\title{
Determination of Redox Potentials for the Watson-Crick Base Pairs, DNA Nucleosides, and Relevant Nucleosides Analogs
}

Carlos E. Crespo-Hernández*,

Department of Chemistry, The Ohio State University, 100 W. $18^{\text {th }}$ Avenue, Columbus, Ohio 43210

David M. Close

Department of Physics, East Tennessee State University, Johnson City, TN 37614

Leonid Gorb ${ }^{\dagger, *}$ and Jerzy Leszczynski ${ }^{\dagger}$

${ }^{\dagger}$ Computational Center for Molecular Modeling Structure and Interactions, Department of Chemistry, Jackson State University, PO Box 17910, 1325 Lynch Str., Jackson, MS 39217; ${ }^{\star}$ Department of Molecular Biophysics, Institute of Molecular Biology and Genetics, National Academy of Science of Ukraine, 150 Zabolotnogo Street, Kyiv 03143, Ukraine

${ }^{\S}$ Current address: Department of Chemistry, Case Western Reserve University, 10900 Euclid Avenue, Cleveland, Ohio 44106-7078

\section{Supporting Information Available}

I. Table 1. Determined Vertical Ionization and Vertical Electron Affinity Energies for Selected Organic Molecules ${ }^{1}$ at B3LYP/6-31++G(d,p) Level of Theory and Their Experimental Reversible Oxidation and Reduction Potentials in DMF or ACN Solutions (including optimized geometries).

\begin{tabular}{|c|c|c|c|c|c|}
\hline No. & Compound/Optimized Geometry & $\mathrm{VIE} / \mathrm{eV}^{\mathrm{b}}$ & $\mathrm{VEA} / \mathrm{eV}^{\mathrm{b}}$ & $\Delta G_{o x}^{\circ} / \mathrm{V}^{\mathrm{c}}$ & $\Delta G_{r e d}^{\circ} / \mathrm{V}^{\mathrm{c}}$ \\
\hline 1 & & & & & \\
& & & & & \\
& & & & & \\
\\
\end{tabular}




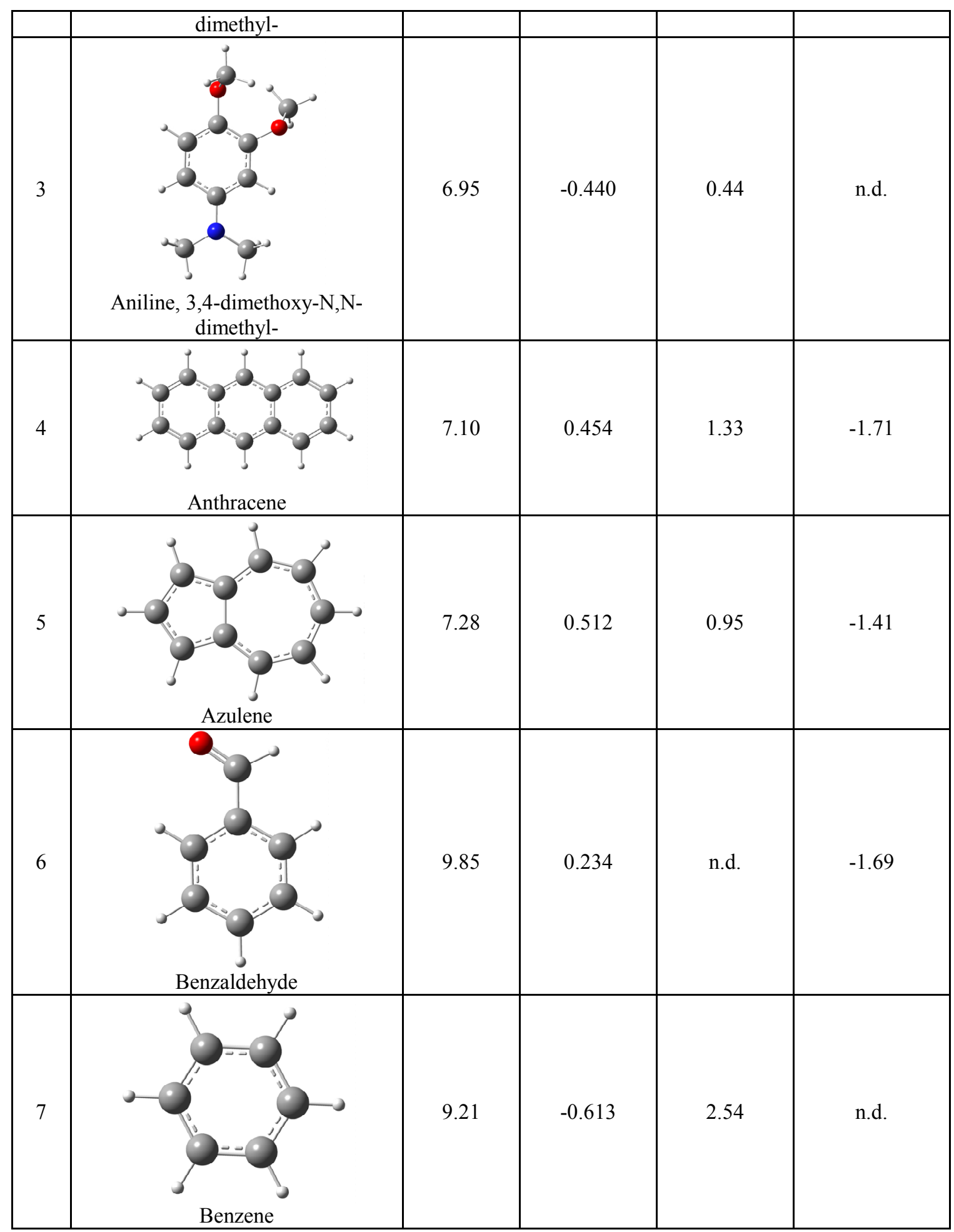




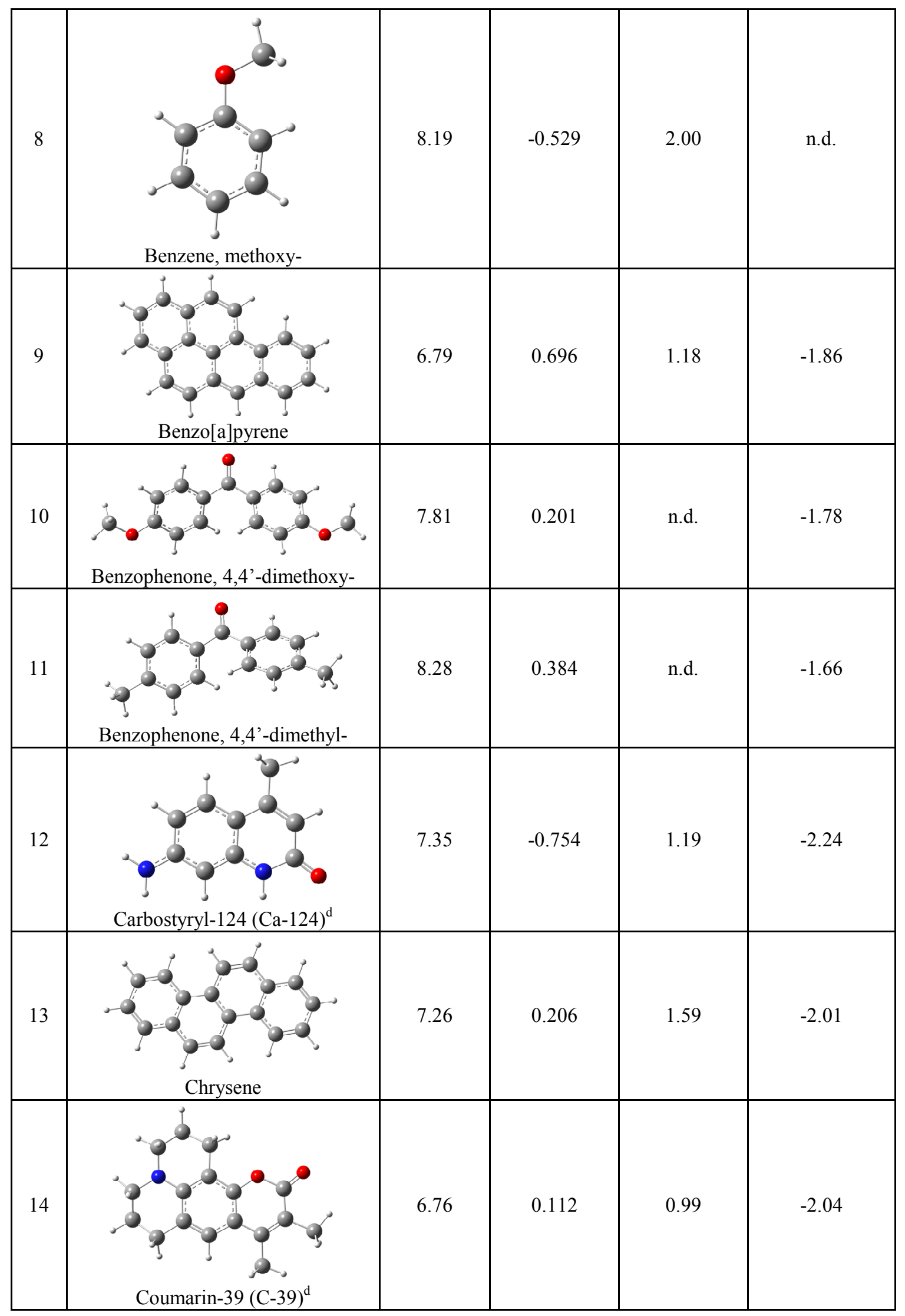




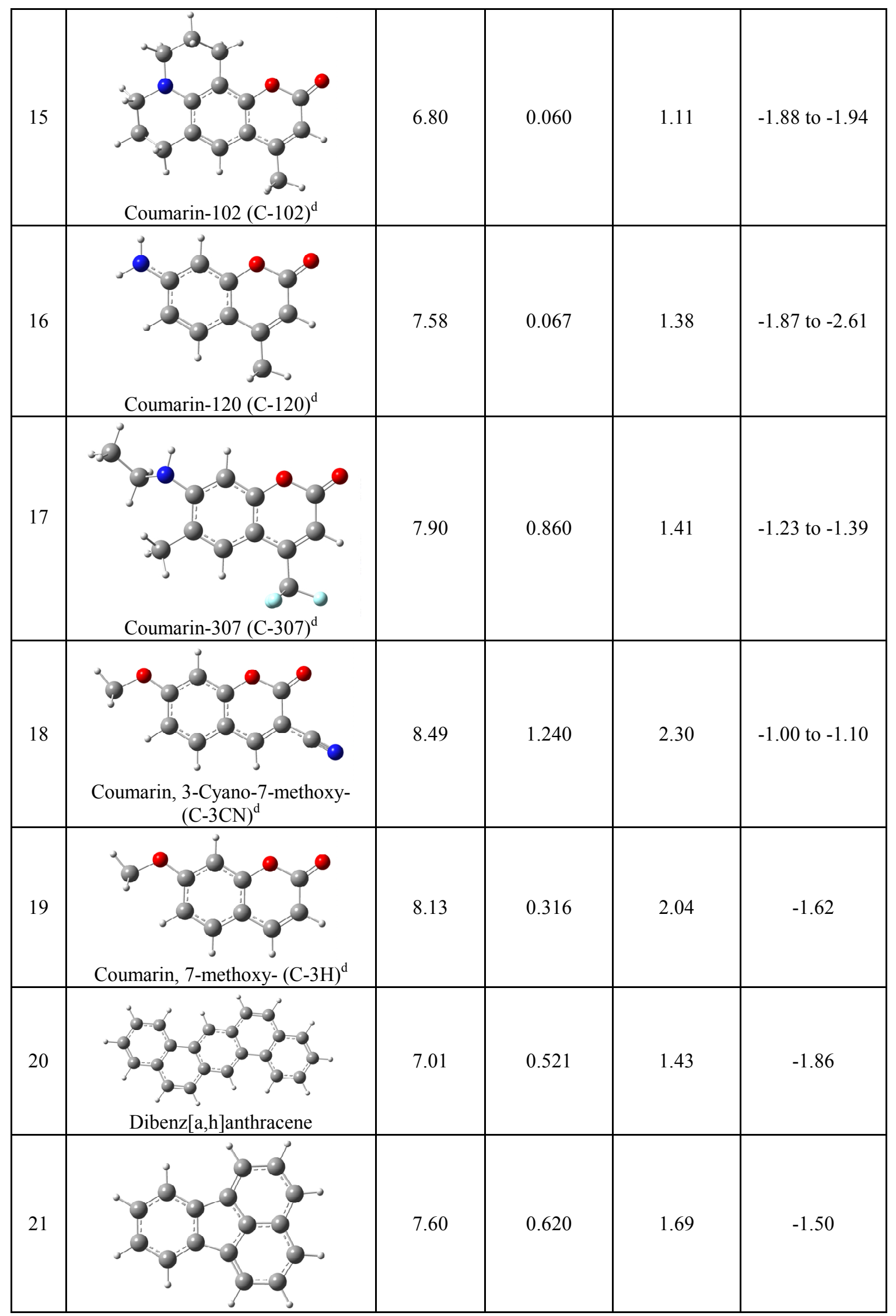




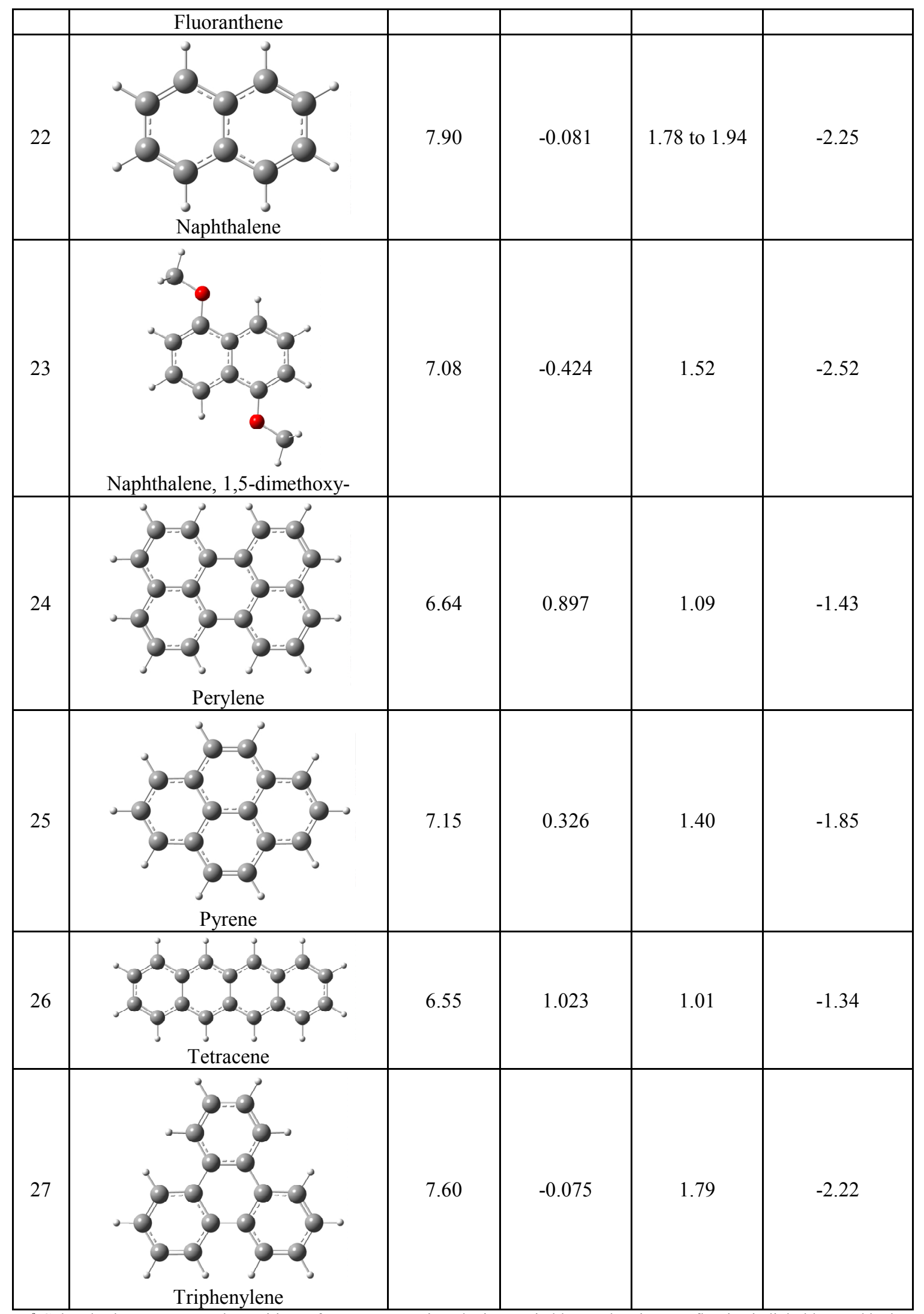

${ }^{a}$ Colored spheres represent the positions of atoms: oxygen in red, nitrogen in blue, carbon in gray, fluorine in light blue, and hydrogen in white. 
${ }^{\mathrm{b}}$ gas phase vertical energies at B3LYP/6-31++G(d,p) level of theory;

c experimental redox potentials are reported versus NHE in DMF or ACN solvents;

d experimental peak potentials taken form Seidel et al. ref. ${ }^{2}$.

II. Table 1S. Electron Affinities for DNA Nucleosides and Nucleosides Analogs

\begin{tabular}{cc}
\hline System $^{\mathrm{a}}$ & $\mathrm{VEA} / \mathrm{eV}^{\mathrm{b}}$ \\
\hline DeoxyAdenosine & 0.386 \\
DeoxyCytidine & -0.024 \\
DeoxyGuanosine & 0.045 \\
DeoxyThymidine & 0.078 \\
DeoxyInosine & 0.047 \\
anti-Deox-2-Aminopurine & 0.071 \\
Deox-2-Aminopurine & $-8.2 \times 10^{-5}$ \\
8-Oxo-DeoxyGuanosine & 0.097 \\
8-Oxo-DeoxyAdenosine & 0.007 \\
5-Methyl-DeoxyCytidine & -0.017 \\
Guanine·Cytosine & -0.002 \\
9-MethylGuanine·1- & -0.051 \\
MethylCytosine & -0.291 \\
Adenine·Thymine & -0.085 \\
9-MethylAdenine· $1-$ & \\
MethylThymine &
\end{tabular}

III. Cartesian Coordinates of the Selected Organic Molecules and Dyes Optimized at B3LYP/631++G(d,p) Level

1. Acenaphthene

\begin{tabular}{|c|c|c|c|c|}
\hline \multirow{2}{*}{$\begin{array}{l}\text { Center } \\
\text { Number }\end{array}$} & \multirow{2}{*}{$\begin{array}{l}\text { Atomic } \\
\text { Number }\end{array}$} & \multirow{2}{*}{$\begin{array}{r}\text { Atomic } \\
\text { Type }\end{array}$} & \multicolumn{2}{|c|}{ Coordinates (Angstroms) } \\
\hline & & & $\begin{array}{ll}\mathrm{X} & \mathrm{Y}\end{array}$ & $\mathrm{Z}$ \\
\hline 1 & 6 & 0.000000 & 0.091504 & -0.000002 \\
\hline 2 & 6 & -1.175751 & 0.875089 & -0.000046 \\
\hline 3 & 6 & 1.175763 & 0.875071 & 0.000142 \\
\hline 4 & 6 & -0.000013 & -1.323752 & -0.000082 \\
\hline 5 & 6 & -0.784948 & 2.344225 & 0.000374 \\
\hline 6 & 6 & -2.398536 & 0.237824 & -0.000259 \\
\hline 7 & 6 & 0.784996 & 2.344214 & -0.000099 \\
\hline 8 & 6 & 2.398537 & 0.237780 & 0.000308 \\
\hline 9 & 6 & 1.276529 & -1.953727 & 0.000083 \\
\hline 10 & 6 & -1.276567 & -1.953703 & -0.000317 \\
\hline 11 & 6 & -2.429725 & -1.186760 & -0.000422 \\
\hline 12 & 6 & 2.429701 & -1.186803 & 0.000292 \\
\hline 13 & 1 & -1.184798 & 2.863706 & -0.878118 \\
\hline 14 & 1 & -1.184475 & 2.863104 & 0.879402 \\
\hline 15 & 1 & -3.332269 & 0.794230 & -0.000253 \\
\hline 16 & 1 & 1.184955 & 2.863613 & 0.878393 \\
\hline 17 & 1 & 1.184467 & 2.863149 & -0.879121 \\
\hline 18 & 1 & 3.332281 & 0.794168 & 0.000388 \\
\hline 19 & 1 & 1.343589 & -3.038581 & 0.000057 \\
\hline 20 & 1 & -1.343646 & -3.038556 & -0.000416 \\
\hline
\end{tabular}




$\begin{array}{lllrrc}21 & 1 & 0 & -3.395374 & -1.685276 & -0.000601 \\ 22 & 1 & 0 & 3.395341 & -1.685336 & 0.000431\end{array}$

2. Aniline, 2,4-dimethoxy-N,N-dimethyl-

\begin{tabular}{|c|c|c|c|c|c|}
\hline \multirow{2}{*}{$\begin{array}{l}\text { Center } \\
\text { Number }\end{array}$} & \multirow{2}{*}{$\begin{array}{l}\text { Atomic } \\
\text { Number }\end{array}$} & \multirow{2}{*}{\multicolumn{2}{|c|}{$\begin{array}{l}\text { Atomic } \\
\text { Type }\end{array}$}} & \multicolumn{2}{|c|}{ Coordinates (Angstroms) } \\
\hline & & & & $\begin{array}{ll}\mathrm{X} & \mathrm{Y}\end{array}$ & $\mathrm{Z}$ \\
\hline 1 & 8 & 0 & 0.752762 & 1.896071 & -0.181420 \\
\hline 2 & 6 & 0 & -0.056075 & 3.059227 & -0.272397 \\
\hline 3 & 6 & 0 & 0.150110 & 0.669819 & -0.081656 \\
\hline 4 & 6 & 0 & 1.014786 & -0.453810 & -0.060395 \\
\hline 5 & 6 & 0 & -1.240406 & 0.515248 & -0.014802 \\
\hline 6 & 7 & 0 & 2.419725 & -0.272569 & -0.162662 \\
\hline 7 & 6 & 0 & 0.410326 & -1.718073 & 0.007836 \\
\hline 8 & 6 & 0 & -1.803545 & -0.767882 & 0.047349 \\
\hline 9 & 6 & 0 & 3.193049 & -1.424170 & -0.598792 \\
\hline 10 & 6 & 0 & 3.055256 & 0.418727 & 0.960946 \\
\hline 11 & 6 & 0 & -0.973893 & -1.888037 & 0.050431 \\
\hline 12 & 8 & 0 & -3.153879 & -1.008592 & 0.103556 \\
\hline 13 & 6 & 0 & -4.045609 & 0.094703 & 0.086377 \\
\hline 14 & 1 & 0 & 0.637992 & 3.894970 & -0.373632 \\
\hline 15 & 1 & 0 & -0.664001 & 3.201153 & 0.630626 \\
\hline 16 & 1 & 0 & -0.711307 & 3.024897 & -1.152175 \\
\hline 17 & 1 & 0 & -1.876305 & 1.388536 & -0.030347 \\
\hline 18 & 1 & 0 & 1.036486 & -2.602801 & 0.026237 \\
\hline 19 & 1 & 0 & 4.205755 & -1.090137 & -0.846852 \\
\hline 20 & 1 & 0 & 2.747336 & -1.859078 & -1.497516 \\
\hline 21 & 1 & 0 & 3.283226 & -2.217719 & 0.167212 \\
\hline 22 & 1 & 0 & 4.075343 & 0.699754 & 0.678213 \\
\hline 23 & 1 & 0 & 3.108848 & -0.217748 & 1.863184 \\
\hline 24 & 1 & 0 & 2.505878 & 1.328133 & 1.202048 \\
\hline 25 & 1 & 0 & -1.415980 & -2.877398 & 0.095575 \\
\hline 26 & 1 & 0 & -5.048758 & -0.331086 & 0.139335 \\
\hline 27 & 1 & 0 & -3.949513 & 0.677284 & -0.839464 \\
\hline 28 & 1 & 0 & -3.888136 & 0.754876 & 0.949716 \\
\hline
\end{tabular}

3. Aniline, 3,4-dimethoxy-N,N-dimethyl-

\begin{tabular}{|c|c|c|c|c|c|}
\hline \multirow{2}{*}{$\begin{array}{l}\text { Center } \\
\text { Number }\end{array}$} & \multirow{2}{*}{$\begin{array}{l}\text { Atomic } \\
\text { Number }\end{array}$} & \multirow{2}{*}{\multicolumn{2}{|c|}{$\begin{array}{l}\text { Atomic } \\
\text { Type }\end{array}$}} & \multicolumn{2}{|c|}{ Coordinates (Angstroms) } \\
\hline & & & & X $\quad Y$ & $\mathrm{Z}$ \\
\hline 1 & 8 & 0 & -1.750525 & 1.597433 & 0.340967 \\
\hline 2 & 6 & 0 & -2.467651 & 2.046135 & -0.818237 \\
\hline 3 & 6 & 0 & -0.862805 & 0.564439 & 0.115949 \\
\hline 4 & 6 & 0 & -1.311190 & -0.732197 & -0.188500 \\
\hline 5 & 6 & 0 & 0.499351 & 0.831948 & 0.247736 \\
\hline 6 & 8 & 0 & -2.656805 & -1.018601 & -0.338306 \\
\hline 7 & 6 & 0 & -0.358218 & -1.727740 & -0.397564 \\
\hline 8 & 6 & 0 & 1.470155 & -0.180162 & 0.073031 \\
\hline 9 & 6 & 0 & -3.383970 & -1.114726 & 0.893518 \\
\hline 10 & 6 & 0 & 1.007055 & -1.468419 & -0.280706 \\
\hline 11 & 7 & 0 & 2.829542 & 0.083932 & 0.253856 \\
\hline 12 & 6 & 0 & 3.799111 & -0.890236 & -0.223190 \\
\hline
\end{tabular}




$\begin{array}{rrrrrr}13 & 6 & 0 & 3.270738 & 1.468265 & 0.325840 \\ 14 & 1 & 0 & -1.771450 & 2.446634 & -1.566123 \\ 15 & 1 & 0 & -3.129255 & 2.843919 & -0.474716 \\ 16 & 1 & 0 & -3.056316 & 1.235167 & -1.257827 \\ 17 & 1 & 0 & 0.772942 & 1.850483 & 0.490675 \\ 18 & 1 & 0 & -0.702813 & -2.723486 & -0.660887 \\ 19 & 1 & 0 & -2.989179 & -1.930813 & 1.512214 \\ 20 & 1 & 0 & -4.419365 & -1.332739 & 0.622523 \\ 21 & 1 & 0 & -3.337329 & -0.174295 & 1.453612 \\ 22 & 1 & 0 & 1.702537 & -2.278499 & -0.458269 \\ 23 & 1 & 0 & 4.803552 & -0.540487 & 0.021867 \\ 24 & 1 & 0 & 3.744307 & -1.054215 & -1.312517 \\ 25 & 1 & 0 & 3.659397 & -1.855371 & 0.274195 \\ 26 & 1 & 0 & 4.347618 & 1.488913 & 0.502766 \\ 27 & 1 & 0 & 2.792516 & 1.986753 & 1.162876 \\ 28 & 1 & 0 & 3.059231 & 2.036009 & -0.595931 \\ --------------------------------------------------\end{array}$

4. Anthracene

\begin{tabular}{|c|c|c|c|c|c|}
\hline \multirow{2}{*}{$\begin{array}{l}\text { Center } \\
\text { Number }\end{array}$} & \multirow{2}{*}{$\begin{array}{l}\text { Atomic } \\
\text { Number }\end{array}$} & \multirow{2}{*}{\multicolumn{2}{|c|}{$\begin{array}{l}\text { Atomic } \\
\text { Type }\end{array}$}} & \multicolumn{2}{|c|}{ Coordinates (Angstroms) } \\
\hline & & & & X $\quad Y$ & $Z$ \\
\hline 1 & 6 & 0 & -1.224817 & -0.722940 & -0.000029 \\
\hline 2 & 6 & 0 & -1.224818 & 0.722932 & -0.000098 \\
\hline 3 & 6 & 0 & 0.000022 & -1.404985 & 0.000080 \\
\hline 4 & 6 & 0 & -2.481167 & -1.408222 & 0.000015 \\
\hline 5 & 6 & 0 & -0.000003 & 1.405014 & -0.000096 \\
\hline 6 & 6 & 0 & -2.481163 & 1.408216 & -0.000171 \\
\hline 7 & 6 & 0 & 1.224831 & -0.722888 & 0.000096 \\
\hline 8 & 6 & 0 & -3.664328 & -0.713480 & -0.000098 \\
\hline 9 & 6 & 0 & 1.224820 & 0.722964 & -0.000011 \\
\hline 10 & 6 & 0 & -3.664328 & 0.713461 & -0.000185 \\
\hline 11 & 6 & 0 & 2.481132 & -1.408233 & 0.000189 \\
\hline 12 & 6 & 0 & 2.481163 & 1.408252 & 0.000018 \\
\hline 13 & 6 & 0 & 3.664325 & -0.713504 & 0.000170 \\
\hline 14 & 6 & 0 & 3.664321 & 0.713440 & 0.000109 \\
\hline 15 & 1 & 0 & 0.000059 & -2.492757 & 0.000167 \\
\hline 16 & 1 & 0 & -2.481014 & -2.495190 & 0.000096 \\
\hline 17 & 1 & 0 & 0.000042 & 2.492791 & -0.000154 \\
\hline 18 & 1 & 0 & -2.480976 & 2.495179 & -0.000240 \\
\hline 19 & 1 & 0 & -4.609518 & -1.248524 & -0.000110 \\
\hline 20 & 1 & 0 & -4.609527 & 1.248472 & -0.000261 \\
\hline 21 & 1 & 0 & 2.480927 & -2.495226 & 0.000264 \\
\hline 22 & 1 & 0 & 2.481029 & 2.495234 & -0.000040 \\
\hline 23 & 1 & 0 & 4.609503 & -1.248550 & 0.000226 \\
\hline 24 & 1 & 0 & 4.609533 & 1.248412 & 0.000120 \\
\hline
\end{tabular}

5. Azulene

\begin{tabular}{|c|c|c|c|c|}
\hline Center & Atomic & Atomic & Coordinate & (Angstrom \\
\hline Number & Number & Type & $\begin{array}{ll}X & Y\end{array}$ & $\mathrm{Z}$ \\
\hline 1 & 6 & -0.554700 & -0.750227 & -0.000100 \\
\hline 2 & 6 & -0.554667 & 0.750167 & 0.000036 \\
\hline
\end{tabular}




$\begin{array}{cccrcc}3 & 6 & 0 & -1.904376 & -1.150954 & 0.000314 \\ 4 & 6 & 0 & 0.552575 & -1.595990 & -0.000120 \\ 5 & 6 & 0 & -1.904191 & 1.151038 & 0.000029 \\ 6 & 6 & 0 & 0.552743 & 1.596017 & 0.000060 \\ 7 & 6 & 0 & -2.711855 & 0.000022 & -0.000250 \\ 8 & 6 & 0 & 1.913202 & -1.267394 & -0.000043 \\ 9 & 6 & 0 & 1.913177 & 1.267403 & 0.000040 \\ 10 & 6 & 0 & 2.506815 & -0.000094 & 0.000003 \\ 11 & 1 & 0 & -2.251115 & -2.177019 & 0.000579 \\ 12 & 1 & 0 & 0.324214 & -2.661242 & -0.000062 \\ 13 & 1 & 0 & -2.250920 & 2.177101 & 0.000046 \\ 14 & 1 & 0 & 0.324452 & 2.661287 & -0.000007 \\ 15 & 1 & 0 & -3.796112 & 0.000107 & -0.000355 \\ 16 & 1 & 0 & 2.601053 & -2.109335 & 0.000008 \\ 17 & 1 & 0 & 2.600900 & 2.109440 & 0.000019 \\ 18 & 1 & 0 & 3.595195 & -0.000261 & -0.000048\end{array}$

6. Benzaldehyde

\begin{tabular}{|c|c|c|c|c|c|}
\hline \multirow{2}{*}{$\begin{array}{l}\text { Center } \\
\text { Number }\end{array}$} & \multirow{2}{*}{\multicolumn{2}{|c|}{$\begin{array}{l}\text { Atomic } \\
\text { Number }\end{array}$}} & & \multicolumn{2}{|c|}{ Coordinates (Angstroms) } \\
\hline & & & & X $\quad Y$ & Z \\
\hline 1 & 6 & 0 & -1.993322 & 0.467172 & 0.000051 \\
\hline 2 & 8 & 0 & -2.855726 & -0.393956 & 0.000034 \\
\hline 3 & 6 & 0 & -0.535509 & 0.207593 & 0.000016 \\
\hline 4 & 6 & 0 & 0.356276 & 1.290275 & 0.000090 \\
\hline 5 & 6 & 0 & -0.039900 & -1.107065 & -0.000085 \\
\hline 6 & 6 & 0 & 1.733930 & 1.065697 & 0.000051 \\
\hline 7 & 6 & 0 & 1.334389 & -1.329771 & -0.000123 \\
\hline 8 & 6 & 0 & 2.221395 & -0.244360 & -0.000055 \\
\hline 9 & 1 & 0 & -2.275156 & 1.542223 & 0.000204 \\
\hline 10 & 1 & 0 & -0.033327 & 2.305780 & 0.000175 \\
\hline 11 & 1 & 0 & -0.746494 & -1.931070 & -0.000139 \\
\hline 12 & 1 & 0 & 2.423697 & 1.904301 & 0.000102 \\
\hline 13 & 1 & 0 & 1.720492 & -2.344796 & -0.000205 \\
\hline 14 & 1 & 0 & 3.293042 & -0.422034 & -0.000087 \\
\hline
\end{tabular}

7. Benzene

\begin{tabular}{|c|c|c|c|c|}
\hline \multirow{2}{*}{$\begin{array}{l}\text { Center } \\
\text { Number }\end{array}$} & \multirow{2}{*}{$\begin{array}{l}\text { Atomic } \\
\text { Number }\end{array}$} & \multirow{2}{*}{$\begin{array}{r}\text { Atomic } \\
\text { Type }\end{array}$} & \multicolumn{2}{|c|}{ Coordinates (Angstroms) } \\
\hline & & & $\begin{array}{ll}X & Y\end{array}$ & Z \\
\hline 1 & 6 & -1.397200 & 0.058100 & 0.000100 \\
\hline 2 & 6 & -0.748900 & -1.180800 & 0.000200 \\
\hline 3 & 6 & -0.648200 & 1.238900 & -0.000100 \\
\hline 4 & 6 & 0.648200 & -1.238900 & 0.000100 \\
\hline 5 & 6 & 0.748900 & 1.180800 & -0.000200 \\
\hline 6 & 6 & 1.397200 & -0.058100 & -0.000100 \\
\hline 7 & 1 & -2.482600 & 0.103300 & 0.000200 \\
\hline 8 & 1 & -1.330600 & -2.098400 & 0.000400 \\
\hline 9 & 1 & -1.151900 & 2.201500 & -0.000200 \\
\hline 10 & 1 & 1.151900 & -2.201500 & 0.000200 \\
\hline 11 & 1 & 1.330600 & 2.098400 & -0.000400 \\
\hline 12 & 1 & 2.482600 & -0.103300 & -0.000200 \\
\hline
\end{tabular}


8. Benzene, methoxy-

\begin{tabular}{|c|c|c|c|c|c|}
\hline \multirow{2}{*}{$\begin{array}{l}\text { Center } \\
\text { Number }\end{array}$} & \multirow{2}{*}{$\begin{array}{l}\text { Atomic } \\
\text { Number }\end{array}$} & \multirow{2}{*}{\multicolumn{2}{|c|}{$\begin{array}{l}\text { Atomic } \\
\text { Type }\end{array}$}} & \multicolumn{2}{|c|}{ Coordinates (Angstroms) } \\
\hline & & & & X $\quad Y$ & $\mathrm{Z}$ \\
\hline 1 & 8 & 0 & -1.762546 & -0.671153 & -0.000094 \\
\hline 2 & 6 & 0 & -2.776634 & 0.325502 & 0.000081 \\
\hline 3 & 6 & 0 & -0.453429 & -0.272093 & -0.000030 \\
\hline 4 & 6 & 0 & 0.496600 & -1.305463 & 0.000004 \\
\hline 5 & 6 & 0 & -0.032776 & 1.063973 & -0.000038 \\
\hline 6 & 6 & 0 & 1.854689 & -1.001061 & 0.000032 \\
\hline 7 & 6 & 0 & 1.337947 & 1.353436 & -0.000011 \\
\hline 8 & 6 & 0 & 2.286716 & 0.331927 & 0.000024 \\
\hline 9 & 1 & 0 & -3.724236 & -0.214675 & 0.000140 \\
\hline 10 & 1 & 0 & -2.718087 & 0.956692 & -0.895580 \\
\hline 11 & 1 & 0 & -2.717895 & 0.956570 & 0.895813 \\
\hline 12 & 1 & 0 & 0.146953 & -2.332861 & 0.000010 \\
\hline 13 & 1 & 0 & -0.749431 & 1.876676 & -0.000069 \\
\hline 14 & 1 & 0 & 2.581117 & -1.808866 & 0.000062 \\
\hline 15 & 1 & 0 & 1.656611 & 2.392154 & -0.000020 \\
\hline 16 & 1 & 0 & 3.346653 & 0.566214 & 0.000037 \\
\hline
\end{tabular}

9. Benzo[a]pyrene

\begin{tabular}{|c|c|c|c|c|c|}
\hline \multirow{2}{*}{$\begin{array}{l}\text { Center } \\
\text { Number }\end{array}$} & \multirow{2}{*}{$\begin{array}{l}\text { Atomic } \\
\text { Number }\end{array}$} & \multirow{2}{*}{\multicolumn{2}{|c|}{$\begin{array}{l}\text { Atomic } \\
\text { Type }\end{array}$}} & \multicolumn{2}{|c|}{ Coordinates (Angstroms } \\
\hline & & & & X $\quad Y$ & $\mathrm{Z}$ \\
\hline 1 & 6 & 0 & 0.313613 & 0.224048 & -0.000026 \\
\hline 2 & 6 & 0 & 1.717348 & -0.056230 & -0.000032 \\
\hline 3 & 6 & 0 & -0.632141 & -0.835135 & 0.000010 \\
\hline 4 & 6 & 0 & -0.129955 & 1.597075 & -0.000084 \\
\hline 5 & 6 & 0 & 2.178588 & -1.406728 & 0.000038 \\
\hline 6 & 6 & 0 & 2.670577 & 1.011415 & -0.000152 \\
\hline 7 & 6 & 0 & -2.043263 & -0.530484 & 0.000029 \\
\hline 8 & 6 & 0 & -0.134866 & -2.179141 & 0.000092 \\
\hline 9 & 6 & 0 & -1.487233 & 1.872528 & -0.000049 \\
\hline 10 & 6 & 0 & 0.858987 & 2.648430 & -0.000173 \\
\hline 11 & 6 & 0 & 1.204788 & -2.453491 & 0.000087 \\
\hline 12 & 6 & 0 & 3.566020 & -1.665239 & -0.000012 \\
\hline 13 & 6 & 0 & 2.189023 & 2.371740 & -0.000179 \\
\hline 14 & 6 & 0 & 4.037873 & 0.708789 & -0.000154 \\
\hline 15 & 6 & 0 & -2.458895 & 0.843163 & 0.000008 \\
\hline 16 & 6 & 0 & -3.055520 & -1.528942 & 0.000164 \\
\hline 17 & 6 & 0 & 4.481027 & -0.619777 & -0.000057 \\
\hline 18 & 6 & 0 & -3.848995 & 1.153035 & 0.000084 \\
\hline 19 & 6 & 0 & -4.395359 & -1.198179 & 0.000177 \\
\hline 20 & 6 & 0 & -4.800218 & 0.158320 & 0.000190 \\
\hline 21 & 1 & 0 & -0.832729 & -3.008032 & 0.000141 \\
\hline 22 & 1 & 0 & -1.822211 & 2.907253 & -0.000068 \\
\hline 23 & 1 & 0 & 0.510443 & 3.677905 & -0.000230 \\
\hline 24 & 1 & 0 & 1.548253 & -3.484689 & 0.000132 \\
\hline 25 & 1 & 0 & 3.910871 & -2.695790 & 0.000040 \\
\hline 26 & 1 & 0 & 2.919122 & 3.176810 & -0.000224 \\
\hline
\end{tabular}




$\begin{array}{rrrrrr}27 & 1 & 0 & 4.759506 & 1.521479 & -0.000192 \\ 28 & 1 & 0 & -2.781147 & -2.577470 & 0.000191 \\ 29 & 1 & 0 & 5.546173 & -0.831779 & -0.000071 \\ 30 & 1 & 0 & -4.145827 & 2.198699 & 0.000045 \\ 31 & 1 & 0 & -5.144105 & -1.984785 & 0.000245 \\ 32 & 1 & 0 & -5.856742 & 0.409222 & 0.000219\end{array}$

10. Benzophenone, 4,4'-dimethoxy-

\begin{tabular}{|c|c|c|c|c|c|}
\hline \multirow{2}{*}{$\begin{array}{l}\text { Center } \\
\text { Number }\end{array}$} & \multirow{2}{*}{$\begin{array}{l}\text { Atomic } \\
\text { Number }\end{array}$} & \multirow{2}{*}{\multicolumn{2}{|c|}{$\begin{array}{l}\text { Atomic } \\
\text { Type }\end{array}$}} & \multicolumn{2}{|c|}{ Coordinates (Angstroms) } \\
\hline & & & & X $\quad Y$ & Z \\
\hline 1 & 6 & 0 & -0.000050 & 1.397719 & 0.000016 \\
\hline 2 & 8 & 0 & -0.000019 & 2.629564 & 0.000246 \\
\hline 3 & 6 & 0 & 1.297249 & 0.654734 & 0.043053 \\
\hline 4 & 6 & 0 & -1.297229 & 0.654696 & -0.043159 \\
\hline 5 & 6 & 0 & 1.446290 & -0.610848 & 0.643095 \\
\hline 6 & 6 & 0 & 2.440707 & 1.284423 & -0.469401 \\
\hline 7 & 6 & 0 & -1.446251 & -0.611015 & -0.642956 \\
\hline 8 & 6 & 0 & -2.440752 & 1.284458 & 0.469148 \\
\hline 9 & 6 & 0 & 2.690399 & -1.223918 & 0.718226 \\
\hline 10 & 6 & 0 & 3.692241 & 0.672991 & -0.421178 \\
\hline 11 & 6 & 0 & -2.690331 & -1.224130 & -0.717923 \\
\hline 12 & 6 & 0 & -3.692267 & 0.673036 & 0.421007 \\
\hline 13 & 6 & 0 & 3.820793 & -0.590493 & 0.176873 \\
\hline 14 & 6 & 0 & -3.820762 & -0.590613 & -0.176729 \\
\hline 15 & 8 & 0 & 4.992649 & -1.276744 & 0.284576 \\
\hline 16 & 8 & 0 & -4.992570 & -1.276908 & -0.284294 \\
\hline 17 & 6 & 0 & 6.183540 & -0.685748 & -0.226648 \\
\hline 18 & 6 & 0 & -6.183614 & -0.685581 & 0.226203 \\
\hline 19 & 1 & 0 & 0.587605 & -1.107587 & 1.082391 \\
\hline 20 & 1 & 0 & 2.334588 & 2.270574 & -0.909737 \\
\hline 21 & 1 & 0 & -0.587598 & -1.107802 & -1.082246 \\
\hline 22 & 1 & 0 & -2.334545 & 2.270672 & 0.909324 \\
\hline 23 & 1 & 0 & 2.813744 & -2.190905 & 1.194737 \\
\hline 24 & 1 & 0 & 4.550613 & 1.183191 & -0.841458 \\
\hline 25 & 1 & 0 & -2.813656 & -2.191244 & -1.194178 \\
\hline 26 & 1 & 0 & -4.550684 & 1.183346 & 0.841067 \\
\hline 27 & 1 & 0 & 6.978855 & -1.404893 & -0.028387 \\
\hline 28 & 1 & 0 & 6.409973 & 0.258526 & 0.283052 \\
\hline 29 & 1 & 0 & 6.109994 & -0.511907 & -1.307034 \\
\hline 30 & 1 & 0 & -6.978940 & -1.404675 & 0.027801 \\
\hline 31 & 1 & 0 & -6.409677 & 0.258566 & -0.283889 \\
\hline 32 & 1 & 0 & -6.110532 & -0.511421 & 1.306570 \\
\hline
\end{tabular}

11. Benzophenone, 4,4'-dimethyl-

\begin{tabular}{lllllc} 
Center & \multicolumn{2}{c}{ Atomic } & Atomic & \multicolumn{2}{c}{ Coordinates (Angstroms) } \\
Number & Number & Type & X & Y & Z \\
- \hdashline 1 & 6 & 0 & -0.000008 & 1.332437 & -0.000025 \\
2 & 8 & 0 & -0.000025 & 2.561955 & 0.000014 \\
3 & 6 & 0 & -1.300390 & 0.587317 & -0.007408 \\
4 & 6 & 0 & 1.300402 & 0.587354 & 0.007347
\end{tabular}




$\begin{array}{cccrrc}5 & 6 & 0 & -1.460486 & -0.663899 & -0.621675 \\ 6 & 6 & 0 & -2.428469 & 1.208473 & 0.556094 \\ 7 & 6 & 0 & 1.460539 & -0.663824 & 0.621735 \\ 8 & 6 & 0 & 2.428450 & 1.208513 & -0.556147 \\ 9 & 6 & 0 & -2.714396 & -1.277222 & -0.664092 \\ 10 & 6 & 0 & -3.669706 & 0.581738 & 0.529653 \\ 11 & 6 & 0 & 2.714452 & -1.277075 & 0.664243 \\ 12 & 6 & 0 & 3.669740 & 0.581826 & -0.529611 \\ 13 & 6 & 0 & -3.836421 & -0.673793 & -0.080636 \\ 14 & 6 & 0 & 3.836480 & -0.673646 & 0.080732 \\ 15 & 6 & 0 & -5.185746 & -1.351663 & -0.093696 \\ 16 & 6 & 0 & 5.185659 & -1.351814 & 0.093533 \\ 17 & 1 & 0 & -0.613244 & -1.149176 & -1.095213 \\ 18 & 1 & 0 & -2.310693 & 2.188243 & 1.007600 \\ 19 & 1 & 0 & 0.613308 & -1.149065 & 1.095331 \\ 20 & 1 & 0 & 2.310675 & 2.188274 & -1.007673 \\ 21 & 1 & 0 & -2.821595 & -2.237854 & -1.161530 \\ 22 & 1 & 0 & -4.527693 & 1.074029 & 0.981391 \\ 23 & 1 & 0 & 2.821696 & -2.237624 & 1.161839 \\ 24 & 1 & 0 & 4.527727 & 1.074149 & -0.981301 \\ 25 & 1 & 0 & -5.982851 & -0.647096 & -0.353670 \\ 26 & 1 & 0 & -5.427334 & -1.763829 & 0.894018 \\ 27 & 1 & 0 & -5.212849 & -2.176010 & -0.811940 \\ 28 & 1 & 0 & 5.983947 & -0.645809 & 0.345723 \\ 29 & 1 & 0 & 5.423411 & -1.770913 & -0.892213 \\ 30 & 1 & 0 & 5.215099 & -2.171301 & 0.817245 \\ -------------------------------\end{array}$

12. Carbostyryl-124 (Ca-124)

\begin{tabular}{|c|c|c|c|c|c|}
\hline \multirow{2}{*}{$\begin{array}{l}\text { Center } \\
\text { Number }\end{array}$} & Atomic & \multicolumn{2}{|c|}{ Atomic } & \multicolumn{2}{|c|}{ Coordinates (Angstroms) } \\
\hline & & & Type & $\begin{array}{ll}\mathrm{X} & \mathrm{Y}\end{array}$ & $\mathrm{Z}$ \\
\hline 1 & 6 & 0 & 1.715285 & 2.669283 & 0.000035 \\
\hline 2 & 6 & 0 & 1.425715 & 1.188839 & -0.000058 \\
\hline 3 & 6 & 0 & 0.053241 & 0.722352 & -0.000050 \\
\hline 4 & 6 & 0 & 2.450178 & 0.286347 & -0.000105 \\
\hline 5 & 6 & 0 & -0.191556 & -0.677124 & 0.000019 \\
\hline 6 & 6 & 0 & -1.074201 & 1.571369 & -0.000009 \\
\hline 7 & 6 & 0 & 2.234029 & -1.152322 & -0.000288 \\
\hline 8 & 7 & 0 & 0.890840 & -1.533415 & -0.000038 \\
\hline 9 & 6 & 0 & -1.497004 & -1.186694 & 0.000071 \\
\hline 10 & 6 & 0 & -2.365931 & 1.078366 & 0.000060 \\
\hline 11 & 8 & 0 & 3.119677 & -2.010726 & 0.000190 \\
\hline 12 & 6 & 0 & -2.595831 & -0.320080 & 0.000102 \\
\hline 13 & 7 & 0 & -3.882427 & -0.808863 & 0.000786 \\
\hline 14 & 1 & 0 & 2.791871 & 2.852981 & 0.000013 \\
\hline 15 & 1 & 0 & 1.286699 & 3.158143 & 0.882777 \\
\hline 16 & 1 & 0 & 1.286639 & 3.158276 & -0.882605 \\
\hline 17 & 1 & 0 & 3.486671 & 0.604873 & -0.000043 \\
\hline 18 & 1 & 0 & -0.928217 & 2.646395 & -0.000035 \\
\hline 19 & 1 & 0 & 0.737365 & -2.534781 & 0.000078 \\
\hline 20 & 1 & 0 & -1.652316 & -2.262926 & 0.000164 \\
\hline 21 & 1 & 0 & -3.211015 & 1.760984 & 0.000179 \\
\hline 22 & 1 & 0 & -4.672129 & -0.186372 & -0.003086 \\
\hline 23 & 1 & 0 & -4.065415 & -1.797845 & -0.002863 \\
\hline
\end{tabular}


13. Chrysene

\begin{tabular}{|c|c|c|c|c|c|}
\hline \multirow{2}{*}{$\begin{array}{l}\text { Center } \\
\text { Number }\end{array}$} & Atomic & \multicolumn{2}{|c|}{ Atomic } & \multicolumn{2}{|c|}{ Coordinates (Angstroms) } \\
\hline & & & Type & $\mathrm{X}$ & $\mathrm{Z}$ \\
\hline 1 & 6 & 0 & -0.429990 & 0.564326 & 0.000016 \\
\hline 2 & 6 & 0 & 0.430003 & -0.564263 & -0.000016 \\
\hline 3 & 6 & 0 & -1.872260 & 0.377238 & 0.000040 \\
\hline 4 & 6 & 0 & 0.152607 & 1.872315 & 0.000072 \\
\hline 5 & 6 & 0 & 1.872246 & -0.377141 & -0.000090 \\
\hline 6 & 6 & 0 & -0.152576 & -1.872265 & 0.000086 \\
\hline 7 & 6 & 0 & -2.404983 & -0.948129 & 0.000137 \\
\hline 8 & 6 & 0 & -2.794919 & 1.456009 & -0.000026 \\
\hline 9 & 6 & 0 & 1.505657 & 2.057001 & 0.000052 \\
\hline 10 & 6 & 0 & 2.405013 & 0.948201 & -0.000037 \\
\hline 11 & 6 & 0 & 2.794830 & -1.455967 & -0.000186 \\
\hline 12 & 6 & 0 & -1.505627 & -2.056920 & 0.000167 \\
\hline 13 & 6 & 0 & -3.808814 & -1.145764 & 0.000191 \\
\hline 14 & 6 & 0 & -4.160828 & 1.239039 & 0.000013 \\
\hline 15 & 6 & 0 & 3.808866 & 1.145673 & -0.000092 \\
\hline 16 & 6 & 0 & 4.160767 & -1.239154 & -0.000230 \\
\hline 17 & 6 & 0 & -4.677989 & -0.073552 & 0.000121 \\
\hline 18 & 6 & 0 & 4.677994 & 0.073411 & -0.000226 \\
\hline 19 & 1 & 0 & -0.488147 & 2.745783 & 0.000172 \\
\hline 20 & 1 & 0 & 0.488156 & -2.745767 & 0.000119 \\
\hline 21 & 1 & 0 & -2.436907 & 2.478725 & -0.000138 \\
\hline 22 & 1 & 0 & 1.918007 & 3.062757 & 0.000137 \\
\hline 23 & 1 & 0 & 2.436827 & -2.478683 & -0.000242 \\
\hline 24 & 1 & 0 & -1.917947 & -3.062687 & 0.000263 \\
\hline 25 & 1 & 0 & -4.190739 & -2.163592 & 0.000272 \\
\hline 26 & 1 & 0 & -4.838991 & 2.087669 & -0.000057 \\
\hline 27 & 1 & 0 & 4.190950 & 2.163436 & -0.000046 \\
\hline 28 & 1 & 0 & 4.838807 & -2.087888 & -0.000320 \\
\hline 29 & 1 & 0 & -5.751787 & -0.236593 & 0.000160 \\
\hline 30 & 1 & 0 & 5.751786 & 0.236490 & -0.000277 \\
\hline
\end{tabular}

14. Coumarin-39 (C-39)

\begin{tabular}{|c|c|c|c|c|}
\hline \multirow{2}{*}{$\begin{array}{l}\text { Center } \\
\text { Number }\end{array}$} & \multirow{2}{*}{$\begin{array}{l}\text { Atomic } \\
\text { Number }\end{array}$} & \multirow{2}{*}{$\begin{array}{l}\text { Atomic } \\
\text { Type }\end{array}$} & \multicolumn{2}{|c|}{ Coordinates (Angstroms } \\
\hline & & & $\begin{array}{ll}X & Y\end{array}$ & Z \\
\hline 1 & 6 & 2.872502 & 2.630131 & 0.016862 \\
\hline 2 & 6 & 2.480950 & 1.173076 & 0.002982 \\
\hline 3 & 6 & 1.070897 & 0.838958 & -0.007793 \\
\hline 4 & 6 & 3.424141 & 0.177572 & 0.000060 \\
\hline 5 & 6 & 0.696300 & -0.515249 & -0.015796 \\
\hline 6 & 6 & 0.020720 & 1.778241 & -0.013175 \\
\hline 7 & 6 & 4.916302 & 0.420151 & 0.010512 \\
\hline 8 & 6 & 3.011809 & -1.227412 & -0.013237 \\
\hline 9 & 6 & -0.630949 & -0.951309 & -0.019429 \\
\hline 10 & 8 & 1.650349 & -1.495870 & -0.021502 \\
\hline 11 & 6 & -1.314825 & 1.408581 & -0.033103 \\
\hline 12 & 8 & 3.756186 & -2.190247 & -0.017850 \\
\hline
\end{tabular}




$\begin{array}{rrrrrr}13 & 6 & 0 & -1.658254 & 0.022677 & -0.028117 \\ 14 & 6 & 0 & -0.934338 & -2.435488 & -0.018999 \\ 15 & 6 & 0 & -2.395442 & 2.472833 & -0.083089 \\ 16 & 7 & 0 & -2.994524 & -0.370843 & 0.003484 \\ 17 & 6 & 0 & -2.375642 & -2.712024 & 0.414393 \\ 18 & 6 & 0 & -3.737881 & 1.936973 & 0.418209 \\ 19 & 6 & 0 & -3.331688 & -1.759313 & -0.296793 \\ 20 & 6 & 0 & -4.042517 & 0.607827 & -0.266599 \\ 21 & 1 & 0 & 3.953459 & 2.764237 & 0.030864 \\ 22 & 1 & 0 & 2.457821 & 3.136430 & 0.896498 \\ 23 & 1 & 0 & 2.479394 & 3.147601 & -0.866276 \\ 24 & 1 & 0 & 0.256084 & 2.838316 & -0.009833 \\ 25 & 1 & 0 & 5.441229 & -0.535511 & 0.002485 \\ 26 & 1 & 0 & 5.232029 & 0.972906 & 0.903036 \\ 27 & 1 & 0 & 5.241255 & 0.993043 & -0.865799 \\ 28 & 1 & 0 & -0.761422 & -2.843774 & -1.024733 \\ 29 & 1 & 0 & -0.227923 & -2.956103 & 0.634252 \\ 30 & 1 & 0 & -2.084595 & 3.344544 & 0.503993 \\ 31 & 1 & 0 & -2.516219 & 2.825733 & -1.117777 \\ 32 & 1 & 0 & -2.651331 & -3.748201 & 0.190894 \\ 33 & 1 & 0 & -2.478339 & -2.570949 & 1.497109 \\ 34 & 1 & 0 & -3.703290 & 1.780332 & 1.503153 \\ 35 & 1 & 0 & -4.542800 & 2.650870 & 0.212408 \\ 36 & 1 & 0 & -4.361486 & -1.929046 & 0.035003 \\ 37 & 1 & 0 & -3.306283 & -1.942283 & -1.386655 \\ 38 & 1 & 0 & -4.164881 & 0.766640 & -1.354095 \\ 39 & 1 & 0 & -4.985826 & 0.192700 & 0.104569 \\ ----------------------------------------------------------\end{array}$

15. Coumarin-102 (C-102)

\begin{tabular}{|c|c|c|c|c|c|}
\hline \multirow{2}{*}{$\begin{array}{l}\text { Center } \\
\text { Number }\end{array}$} & \multirow{2}{*}{$\begin{array}{l}\text { Atomic } \\
\text { Number }\end{array}$} & \multirow{2}{*}{\multicolumn{2}{|c|}{$\begin{array}{c}\text { Atomic } \\
\text { Type }\end{array}$}} & \multicolumn{2}{|c|}{ Coordinates (Angstroms } \\
\hline & & & & X $\quad Y$ & $\mathrm{Z}$ \\
\hline 1 & 6 & 0 & -3.117932 & 2.749297 & 0.143321 \\
\hline 2 & 6 & 0 & -2.728535 & 1.296638 & 0.052513 \\
\hline 3 & 6 & 0 & -1.335119 & 0.914344 & 0.054119 \\
\hline 4 & 6 & 0 & -3.682949 & 0.324974 & -0.030577 \\
\hline 5 & 6 & 0 & -1.014011 & -0.456675 & -0.030791 \\
\hline 6 & 6 & 0 & -0.253436 & 1.814740 & 0.135442 \\
\hline 7 & 6 & 0 & -3.359454 & -1.083733 & -0.116905 \\
\hline 8 & 6 & 0 & 0.297832 & -0.933855 & -0.048962 \\
\hline 9 & 8 & 0 & -2.000326 & -1.403855 & -0.115518 \\
\hline 10 & 6 & 0 & 1.064863 & 1.395956 & 0.140680 \\
\hline 11 & 8 & 0 & -4.147301 & -2.005638 & -0.190707 \\
\hline 12 & 6 & 0 & & $0.001 \mathrm{C}$ & 0.030854 \\
\hline 13 & 6 & 0 & 0.587189 & -2.415006 & -0.133435 \\
\hline 14 & 6 & 0 & 2.206039 & 2.381368 & 0.244005 \\
\hline 15 & 7 & 0 & 2.672537 & -0.427982 & 0.008007 \\
\hline 16 & 6 & 0 & 1.921054 & -2.727236 & 0.549512 \\
\hline 17 & 6 & 0 & 3.400218 & 1.879922 & -0.572800 \\
\hline 18 & 6 & 0 & 3.026114 & -1.846153 & -0.023241 \\
\hline 19 & 6 & 0 & 3.806300 & 0.491903 & -0.085709 \\
\hline 20 & 1 & 0 & -4.204154 & 2.862715 & 0.129668 \\
\hline 21 & 1 & 0 & -2.703290 & 3.322500 & -0.694043 \\
\hline 22 & 1 & 0 & -2.737928 & 3.202973 & 1.066112 \\
\hline
\end{tabular}




\begin{tabular}{|c|c|c|c|c|c|}
\hline 23 & 1 & 0 & -4.740855 & 0.560421 & -0.033807 \\
\hline 24 & 1 & 0 & -0.454441 & 2.880094 & 0.201545 \\
\hline 25 & 1 & 0 & -0.228409 & -2.979611 & 0.324926 \\
\hline 26 & 1 & 0 & 0.625591 & -2.734731 & -1.185184 \\
\hline 27 & 1 & 0 & 1.881590 & 3.369255 & -0.100278 \\
\hline 28 & 1 & 0 & 2.515248 & 2.499865 & 1.293548 \\
\hline 29 & 1 & 0 & 1.833617 & -2.548624 & 1.628359 \\
\hline 30 & 1 & 0 & 2.193456 & -3.779178 & 0.413561 \\
\hline 31 & 1 & 0 & 3.126077 & 1.834620 & -1.633946 \\
\hline 32 & 1 & 0 & 4.257808 & 2.555234 & -0.482699 \\
\hline 33 & 1 & 0 & 3.254597 & -2.151933 & -1.058474 \\
\hline 34 & 1 & 0 & 3.949323 & -1.973921 & 0.556595 \\
\hline 35 & 1 & 0 & 4.302325 & 0.568938 & 0.896117 \\
\hline & 1 & 0 & 4.539485 & 0.054390 & -0.776399 \\
\hline
\end{tabular}

16. Coumarin-120 (C-120)

\begin{tabular}{|c|c|c|c|c|c|}
\hline \multirow{2}{*}{$\begin{array}{l}\text { Center } \\
\text { Number }\end{array}$} & Atomic & \multicolumn{2}{|c|}{ Atomic } & \multicolumn{2}{|c|}{ Coordinates (Angstroms) } \\
\hline & Numbe & & Type & $\mathrm{X}$ & $\mathrm{Z}$ \\
\hline 1 & 6 & 0 & -1.779556 & 2.642590 & 0.000269 \\
\hline 2 & 6 & 0 & -1.451265 & 1.172885 & 0.000120 \\
\hline 3 & 6 & 0 & -0.071965 & 0.732877 & 0.000077 \\
\hline 4 & 6 & 0 & -2.440470 & 0.235548 & 0.000113 \\
\hline 5 & 6 & 0 & 0.186305 & -0.655651 & -0.000091 \\
\hline 6 & 6 & 0 & 1.050494 & 1.588206 & 0.000147 \\
\hline 7 & 6 & 0 & -2.175292 & -1.190700 & -0.000063 \\
\hline 8 & 8 & 0 & -0.829108 & -1.567942 & -0.000202 \\
\hline 9 & 6 & 0 & 1.477791 & -1.173060 & -0.000133 \\
\hline 10 & 6 & 0 & 2.343330 & 1.095688 & 0.000104 \\
\hline 11 & 8 & 0 & -3.001982 & -2.078391 & -0.000254 \\
\hline 12 & 6 & 0 & 2.577333 & -0.301716 & -0.000035 \\
\hline 13 & 7 & 0 & 3.861329 & -0.791495 & -0.000252 \\
\hline 14 & 1 & 0 & -2.860178 & 2.799997 & 0.000275 \\
\hline 15 & 1 & 0 & -1.360940 & 3.140399 & -0.882106 \\
\hline 16 & 1 & 0 & -1.360956 & 3.140217 & 0.882755 \\
\hline 17 & 1 & 0 & -3.488855 & 0.510372 & 0.000208 \\
\hline 18 & 1 & 0 & 0.900490 & 2.662818 & 0.000275 \\
\hline 19 & 1 & 0 & 1.609553 & -2.250244 & -0.000280 \\
\hline 20 & 1 & 0 & 3.186492 & 1.780580 & 0.000165 \\
\hline 21 & 1 & 0 & 4.653254 & -0.171639 & 0.000644 \\
\hline 22 & 1 & 0 & 4.040325 & -1.781377 & 0.000434 \\
\hline
\end{tabular}

17. Coumarin-307 (C-307)

\begin{tabular}{|c|c|c|c|c|}
\hline \multirow{2}{*}{$\begin{array}{l}\text { Center } \\
\text { Number }\end{array}$} & \multirow{2}{*}{$\begin{array}{l}\text { Atomic } \\
\text { Number }\end{array}$} & \multirow{2}{*}{$\begin{array}{r}\text { Atomic } \\
\text { Type }\end{array}$} & \multicolumn{2}{|c|}{ Coordinates (Angstroms) } \\
\hline & & & X $\quad Y$ & Z \\
\hline 1 & 6 & -2.806715 & -1.363490 & 0.098428 \\
\hline 2 & 9 & -4.141757 & -1.206644 & 0.211007 \\
\hline 3 & 9 & -2.586747 & -2.108124 & -1.015407 \\
\hline 4 & 9 & -2.404111 & -2.109721 & 1.158541 \\
\hline 5 & 6 & -2.066776 & -0.043260 & 0.035686 \\
\hline 6 & 6 & -0.623931 & -0.025701 & -0.061460 \\
\hline
\end{tabular}




$\begin{array}{cccrrr}7 & 6 & 0 & -2.776596 & 1.112793 & 0.072327 \\ 8 & 6 & 0 & -0.011007 & 1.241340 & -0.121742 \\ 9 & 6 & 0 & 0.224750 & -1.152321 & -0.116523 \\ 10 & 6 & 0 & -2.123246 & 2.414852 & 0.012794 \\ 11 & 6 & 0 & 1.369387 & 1.374224 & -0.197887 \\ 12 & 8 & 0 & -0.738220 & 2.400461 & -0.087211 \\ 13 & 6 & 0 & 1.607503 & -1.055771 & -0.212016 \\ 14 & 8 & 0 & -2.684064 & 3.487639 & 0.042430 \\ 15 & 6 & 0 & 2.198240 & 0.243125 & -0.233058 \\ 16 & 6 & 0 & 2.428612 & -2.313864 & -0.372165 \\ 17 & 7 & 0 & 3.582620 & 0.424446 & -0.368986 \\ 18 & 6 & 0 & 4.521925 & -0.089892 & 0.644279 \\ 19 & 6 & 0 & 5.955595 & 0.272655 & 0.265949 \\ 20 & 1 & 0 & -3.855480 & 1.129990 & 0.146685 \\ 21 & 1 & 0 & -0.216446 & -2.142387 & -0.112696 \\ 22 & 1 & 0 & 1.792062 & 2.374064 & -0.213654 \\ 23 & 1 & 0 & 3.244804 & -2.158436 & -1.084950 \\ 24 & 1 & 0 & 1.802786 & -3.129854 & -0.743380 \\ 25 & 1 & 0 & 2.870842 & -2.650500 & 0.573181 \\ 26 & 1 & 0 & 3.795246 & 1.396439 & -0.569809 \\ 27 & 1 & 0 & 4.280583 & 0.314347 & 1.640556 \\ 28 & 1 & 0 & 4.424133 & -1.174461 & 0.707668 \\ 29 & 1 & 0 & 6.653431 & -0.104593 & 1.019725 \\ 30 & 1 & 0 & 6.090330 & 1.359418 & 0.206472 \\ 31 & 1 & 0 & 6.224739 & -0.157689 & -0.703585 \\ ---------------------------------\end{array}$

18. Coumarin, 3-Cyano-7-methoxy- (C-3CN)

\begin{tabular}{|c|c|c|c|c|c|}
\hline \multirow{2}{*}{$\begin{array}{l}\text { Center } \\
\text { Number }\end{array}$} & \multirow{2}{*}{$\begin{array}{l}\text { Atomic } \\
\text { Number }\end{array}$} & \multirow{2}{*}{\multicolumn{2}{|c|}{$\begin{array}{l}\text { Atomic } \\
\text { Type }\end{array}$}} & \multicolumn{2}{|c|}{ Coordinates (Angstroms } \\
\hline & & & & X $\quad Y$ & $\mathrm{Z}$ \\
\hline 1 & 6 & 0 & 3.824571 & -0.756296 & -0.000148 \\
\hline 2 & 7 & 0 & 4.925747 & -1.132892 & 0.000876 \\
\hline 3 & 6 & 0 & 2.457939 & -0.340666 & -0.000227 \\
\hline 4 & 6 & 0 & 1.437189 & -1.251773 & -0.000227 \\
\hline 5 & 6 & 0 & 2.184527 & 1.106624 & -0.000212 \\
\hline 6 & 6 & 0 & 0.074824 & -0.828447 & -0.000110 \\
\hline 7 & 8 & 0 & 3.007360 & 1.987558 & -0.000255 \\
\hline 8 & 8 & 0 & 0.838093 & 1.465136 & -0.000178 \\
\hline 9 & 6 & 0 & -0.185594 & 0.561974 & -0.000081 \\
\hline 10 & 6 & 0 & -1.027401 & -1.705424 & -0.000028 \\
\hline 11 & 6 & 0 & -1.477979 & 1.064009 & 0.000001 \\
\hline 12 & 6 & 0 & -2.328843 & -1.227392 & 0.000076 \\
\hline 13 & 6 & 0 & -2.554961 & 0.167283 & 0.000081 \\
\hline 14 & 8 & 0 & -3.783171 & 0.736637 & 0.000195 \\
\hline 15 & 6 & 0 & -4.943237 & -0.096400 & 0.000133 \\
\hline 16 & 1 & 0 & 1.664585 & -2.314279 & -0.000269 \\
\hline 17 & 1 & 0 & -0.847389 & -2.776650 & -0.000032 \\
\hline 18 & 1 & 0 & -1.655688 & 2.132813 & 0.000011 \\
\hline 19 & 1 & 0 & -3.155727 & -1.925669 & 0.000155 \\
\hline 20 & 1 & 0 & -5.792042 & 0.587119 & 0.000097 \\
\hline 21 & 1 & 0 & -4.979258 & -0.724343 & 0.897616 \\
\hline 22 & 1 & 0 & -4.979171 & -0.724332 & -0.897359 \\
\hline
\end{tabular}


19. Coumarin, 7-methoxy- (C-3H)

\begin{tabular}{|c|c|c|c|c|c|}
\hline \multirow{2}{*}{$\begin{array}{l}\text { Center } \\
\text { Number }\end{array}$} & \multirow{2}{*}{$\begin{array}{l}\text { Atomic } \\
\text { Number }\end{array}$} & \multirow{2}{*}{\multicolumn{2}{|c|}{$\begin{array}{l}\text { Atomic } \\
\text { Type }\end{array}$}} & \multicolumn{2}{|c|}{ Coordinates (Angstroms) } \\
\hline & & & & X $\quad Y$ & Z \\
\hline 1 & 8 & 0 & 3.098002 & -1.021049 & -0.000013 \\
\hline 2 & 6 & 0 & 4.349378 & -0.338934 & 0.000046 \\
\hline 3 & 6 & 0 & 1.949244 & -0.294285 & -0.000011 \\
\hline 4 & 6 & 0 & 0.765443 & -1.041838 & -0.000016 \\
\hline 5 & 6 & 0 & 1.905413 & 1.115096 & -0.000010 \\
\hline 6 & 6 & 0 & -0.453607 & -0.376610 & -0.000022 \\
\hline 7 & 6 & 0 & 0.672741 & 1.757084 & -0.000005 \\
\hline 8 & 6 & 0 & -0.531980 & 1.034160 & -0.000012 \\
\hline 9 & 8 & 0 & -1.584389 & -1.144114 & -0.000036 \\
\hline 10 & 6 & 0 & -1.841639 & 1.628283 & 0.000018 \\
\hline 11 & 6 & 0 & -2.871493 & -0.599479 & -0.000124 \\
\hline 12 & 6 & 0 & -2.955475 & 0.856060 & 0.000003 \\
\hline 13 & 8 & 0 & -3.805686 & -1.370167 & 0.000110 \\
\hline 14 & 1 & 0 & 5.109625 & -1.120232 & 0.000084 \\
\hline 15 & 1 & 0 & 4.464252 & 0.281540 & 0.896716 \\
\hline 16 & 1 & 0 & 4.464337 & 0.281536 & -0.896616 \\
\hline 17 & 1 & 0 & 0.801955 & -2.124754 & -0.000021 \\
\hline 18 & 1 & 0 & 2.814713 & 1.702438 & -0.000007 \\
\hline 19 & 1 & 0 & 0.634411 & 2.842998 & 0.000005 \\
\hline 20 & 1 & 0 & -1.924220 & 2.712594 & 0.000069 \\
\hline 21 & 1 & 0 & -3.956636 & 1.269297 & 0.000066 \\
\hline
\end{tabular}

20. Dibenz[a,h]anthracene

\begin{tabular}{|c|c|c|c|c|c|}
\hline \multirow{2}{*}{$\begin{array}{l}\text { Center } \\
\text { Number }\end{array}$} & \multirow{2}{*}{$\begin{array}{l}\text { Atomic } \\
\text { Number }\end{array}$} & \multirow{2}{*}{\multicolumn{2}{|c|}{$\begin{array}{l}\text { Atomic } \\
\text { Type }\end{array}$}} & \multicolumn{2}{|c|}{ Coordinates (Angstroms) } \\
\hline & & & & X $\quad Y$ & $\mathrm{Z}$ \\
\hline 1 & 6 & 0 & -1.425680 & 0.177957 & -0.000051 \\
\hline 2 & 6 & 0 & -2.882763 & 0.306072 & -0.000116 \\
\hline 3 & 6 & 0 & -0.839155 & -1.131746 & 0.000006 \\
\hline 4 & 6 & 0 & -0.556131 & 1.273817 & -0.000056 \\
\hline 5 & 6 & 0 & -3.681748 & -0.875391 & -0.000099 \\
\hline 6 & 6 & 0 & -3.545459 & 1.554816 & -0.000193 \\
\hline 7 & 6 & 0 & 0.556165 & -1.273782 & 0.000058 \\
\hline 8 & 6 & 0 & -1.692831 & -2.290177 & 0.000013 \\
\hline 9 & 6 & 0 & 0.839177 & 1.131803 & -0.000007 \\
\hline 10 & 6 & 0 & 3.046823 & -2.167911 & -0.000027 \\
\hline 11 & 6 & 0 & -5.091499 & -0.765882 & -0.000143 \\
\hline 12 & 6 & 0 & -4.929305 & 1.639677 & -0.000239 \\
\hline 13 & 6 & 0 & 1.425666 & -0.177905 & 0.000056 \\
\hline 14 & 6 & 0 & 1.692864 & 2.290232 & -0.000018 \\
\hline 15 & 6 & 0 & -5.712832 & 0.470893 & -0.000213 \\
\hline 16 & 6 & 0 & 2.882747 & -0.306061 & 0.000114 \\
\hline 17 & 6 & 0 & 3.046855 & 2.167915 & 0.000019 \\
\hline 18 & 6 & 0 & 3.681766 & 0.875387 & 0.000085 \\
\hline 19 & 6 & 0 & 3.545392 & -1.554809 & 0.000202 \\
\hline 20 & 6 & 0 & 5.091503 & 0.765818 & 0.000136 \\
\hline 21 & 6 & 0 & 4.929274 & -1.639719 & 0.000251 \\
\hline 22 & 6 & 0 & 5.712822 & -0.470983 & 0.000217 \\
\hline 23 & 1 & 0 & -0.951758 & 2.284075 & -0.000098 \\
\hline
\end{tabular}




\begin{tabular}{|c|c|c|c|c|c|}
\hline 24 & 1 & 0 & -2.969884 & 2.473709 & -0.000220 \\
\hline 25 & 1 & 0 & 0.951747 & -2.284054 & 0.000099 \\
\hline 26 & 1 & 0 & -1.229018 & -3.273073 & 0.000060 \\
\hline 27 & 1 & 0 & -3.678844 & -3.052092 & -0.000012 \\
\hline 28 & 1 & 0 & -5.684841 & -1.676715 & -0.000125 \\
\hline 29 & 1 & 0 & -5.408287 & 2.614433 & -0.000295 \\
\hline 30 & 1 & 0 & 1.229066 & 3.273152 & -0.000059 \\
\hline 31 & 1 & 0 & -6.796575 & 0.539874 & -0.000249 \\
\hline 32 & 1 & 0 & 3.678889 & 3.052087 & 0.000002 \\
\hline 33 & 1 & 0 & 2.969806 & -2.473685 & 0.000238 \\
\hline 34 & 1 & 0 & 5.684912 & 1.676614 & 0.000111 \\
\hline 35 & 1 & 0 & 5.408182 & -2.614524 & 0.000320 \\
\hline 36 & 1 & 0 & 6.796582 & -0.539918 & 0.000258 \\
\hline
\end{tabular}

21. Fluoranthene

\begin{tabular}{|c|c|c|c|c|c|}
\hline \multirow{2}{*}{$\begin{array}{l}\text { Center } \\
\text { Number }\end{array}$} & \multirow{2}{*}{\multicolumn{2}{|c|}{$\begin{array}{l}\text { Atomic } \\
\text { Number }\end{array}$}} & & \multicolumn{2}{|c|}{ Coordinates (Angstroms) } \\
\hline & & & & X $\quad Y$ & $\mathrm{Z}$ \\
\hline 1 & 6 & 0 & 0.904687 & 0.000007 & -0.000171 \\
\hline 2 & 6 & 0 & 0.105519 & -1.172203 & 0.000064 \\
\hline 3 & 6 & 0 & 0.105499 & 1.172202 & -0.000132 \\
\hline 4 & 6 & 0 & 2.308903 & 0.000014 & -0.000375 \\
\hline 5 & 6 & 0 & -1.298260 & -0.714102 & 0.000129 \\
\hline 6 & 6 & 0 & 0.740862 & -2.399025 & 0.000106 \\
\hline 7 & 6 & 0 & -1.298275 & 0.714094 & 0.000261 \\
\hline 8 & 6 & 0 & 0.740820 & 2.399018 & -0.000332 \\
\hline 9 & 6 & 0 & 2.936618 & 1.279929 & -0.000588 \\
\hline 10 & 6 & 0 & 2.936634 & -1.279899 & -0.000322 \\
\hline 11 & 6 & 0 & -2.501220 & -1.415910 & 0.000269 \\
\hline 12 & 6 & 0 & 2.165075 & -2.432020 & -0.000122 \\
\hline 13 & 6 & 0 & -2.501244 & 1.415899 & 0.000520 \\
\hline 14 & 6 & 0 & 2.165043 & 2.432035 & -0.000540 \\
\hline 15 & 6 & 0 & -3.706203 & -0.699966 & 0.000542 \\
\hline 16 & 6 & 0 & -3.706214 & 0.699938 & 0.000650 \\
\hline 17 & 1 & 0 & 0.185124 & -3.332824 & 0.000241 \\
\hline 18 & 1 & 0 & 0.185117 & 3.332823 & -0.000268 \\
\hline 19 & 1 & 0 & 4.021030 & 1.352638 & -0.000742 \\
\hline 20 & 1 & 0 & 4.021042 & -1.352677 & -0.000487 \\
\hline 21 & 1 & 0 & -2.511714 & -2.502519 & 0.000253 \\
\hline 22 & 1 & 0 & 2.661023 & -3.398549 & -0.000133 \\
\hline 23 & 1 & 0 & -2.511751 & 2.502520 & 0.000528 \\
\hline 24 & 1 & 0 & 2.661005 & 3.398562 & -0.000653 \\
\hline 25 & 1 & 0 & -4.650165 & -1.237153 & 0.000688 \\
\hline 26 & 1 & 0 & -4.650167 & 1.237112 & 0.000821 \\
\hline
\end{tabular}

22. Naphthalene

\begin{tabular}{llllll} 
Center & \multicolumn{2}{c}{ Atomic } & Atomic & \multicolumn{3}{c}{ Coordinates (Angstroms) } \\
Number & Number & Type & X & Y & Z \\
- \hdashline 1 & 6 & 0 & 0.000007 & -0.717456 & -0.000006 \\
2 & 6 & 0 & 0.000016 & 0.717460 & 0.000006 \\
3 & 6 & 0 & 1.245862 & -1.403944 & -0.000020
\end{tabular}




$\begin{array}{cccrrr}4 & 6 & 0 & -1.245886 & -1.403942 & 0.000013 \\ 5 & 6 & 0 & -1.245874 & 1.403950 & 0.000026 \\ 6 & 6 & 0 & 1.245880 & 1.403950 & -0.000005 \\ 7 & 6 & 0 & 2.436200 & -0.709013 & 0.000018 \\ 8 & 6 & 0 & -2.436208 & -0.709017 & 0.000024 \\ 9 & 6 & 0 & -2.436205 & 0.709019 & -0.000039 \\ 10 & 6 & 0 & 2.436213 & 0.708998 & -0.000019 \\ 11 & 1 & 0 & 1.244934 & -2.490962 & -0.000017 \\ 12 & 1 & 0 & -1.244935 & -2.490954 & -0.000011 \\ 13 & 1 & 0 & -1.244915 & 2.490960 & 0.000030 \\ 14 & 1 & 0 & 1.244928 & 2.490969 & 0.000028 \\ 15 & 1 & 0 & 3.379692 & -1.247222 & 0.000023 \\ 16 & 1 & 0 & -3.379728 & -1.247195 & -0.000005 \\ 17 & 1 & 0 & -3.379721 & 1.247198 & -0.000051 \\ 18 & 1 & 0 & 3.379713 & 1.247173 & 0.000010 \\ ----------------------------------------------------------\end{array}$

23. Naphthalene, 1,5-dimethoxy-

\begin{tabular}{|c|c|c|c|c|c|}
\hline \multirow{2}{*}{$\begin{array}{l}\text { Center } \\
\text { Number }\end{array}$} & Atomic & \multicolumn{2}{|c|}{ Atomic } & \multicolumn{2}{|c|}{ Coordinates (Angstroms) } \\
\hline & Numbe & & Type & $\mathrm{X}$ & $\mathrm{Z}$ \\
\hline 1 & 8 & 0 & -2.876049 & 0.854368 & 0.000144 \\
\hline 2 & 6 & 0 & -4.224943 & 0.407377 & 0.000430 \\
\hline 3 & 6 & 0 & -1.877864 & -0.079835 & 0.000114 \\
\hline 4 & 6 & 0 & -0.548703 & 0.457922 & 0.000004 \\
\hline 5 & 6 & 0 & -2.090334 & -1.446153 & 0.000202 \\
\hline 6 & 6 & 0 & 0.548702 & -0.457917 & -0.000030 \\
\hline 7 & 6 & 0 & -0.308006 & 1.858790 & -0.000085 \\
\hline 8 & 6 & 0 & -0.984642 & -2.331322 & 0.000178 \\
\hline 9 & 6 & 0 & 1.877865 & 0.079838 & -0.000152 \\
\hline 10 & 6 & 0 & 0.308006 & -1.858785 & 0.000059 \\
\hline 11 & 6 & 0 & 0.984642 & 2.331326 & -0.000198 \\
\hline 12 & 8 & 0 & 2.876046 & -0.854368 & -0.000143 \\
\hline 13 & 6 & 0 & 2.090334 & 1.446156 & -0.000236 \\
\hline 14 & 6 & 0 & 4.224944 & -0.407389 & -0.000335 \\
\hline 15 & 1 & 0 & -4.837360 & 1.310044 & 0.000530 \\
\hline 16 & 1 & 0 & -4.448452 & -0.185782 & 0.896121 \\
\hline 17 & 1 & 0 & -4.448827 & -0.185825 & -0.895142 \\
\hline 18 & 1 & 0 & -3.093463 & -1.854810 & 0.000284 \\
\hline 19 & 1 & 0 & -1.150405 & 2.539335 & -0.000064 \\
\hline 20 & 1 & 0 & -1.171476 & -3.401329 & 0.000254 \\
\hline 21 & 1 & 0 & 1.150405 & -2.539330 & 0.000046 \\
\hline 22 & 1 & 0 & 1.171478 & 3.401332 & -0.000267 \\
\hline 23 & 1 & 0 & 3.093462 & 1.854814 & -0.000322 \\
\hline 24 & 1 & 0 & 4.837353 & -1.310061 & -0.000368 \\
\hline 25 & 1 & 0 & 4.448764 & 0.185833 & 0.895239 \\
\hline 26 & 1 & 0 & 4.448529 & 0.185746 & -0.896024 \\
\hline
\end{tabular}

24. Perylene

\begin{tabular}{|c|c|c|c|c|}
\hline Center & Atomic & Atomic & Coordinate & es (Angstroms) \\
\hline Number & Number & Type & $\mathrm{X} \quad \mathrm{Y}$ & $\mathrm{Z}$ \\
\hline 1 & 6 & 1.440447 & 0.000012 & 0.000014 \\
\hline
\end{tabular}




$\begin{array}{cccccc}2 & 6 & 0 & 0.738852 & -1.250295 & 0.000068 \\ 3 & 6 & 0 & 0.738820 & 1.250294 & -0.000029 \\ 4 & 6 & 0 & 2.876882 & 0.000003 & 0.000050 \\ 5 & 6 & 0 & -0.738848 & -1.250306 & -0.000018 \\ 6 & 6 & 0 & 1.479943 & -2.429441 & 0.000198 \\ 7 & 6 & 0 & -0.738868 & 1.250319 & -0.000010 \\ 8 & 6 & 0 & 1.479889 & 2.429450 & -0.000071 \\ 9 & 6 & 0 & 3.578341 & 1.233593 & 0.000001 \\ 10 & 6 & 0 & 3.578389 & -1.233556 & 0.000157 \\ 11 & 6 & 0 & -1.440442 & -0.000018 & -0.000033 \\ 12 & 6 & 0 & -1.479889 & -2.429509 & -0.000074 \\ 13 & 6 & 0 & 2.887630 & -2.425016 & 0.000223 \\ 14 & 6 & 0 & -1.479911 & 2.429491 & 0.000017 \\ 15 & 6 & 0 & 2.887567 & 2.425037 & -0.000060 \\ 16 & 6 & 0 & -2.876901 & -0.000028 & -0.000101 \\ 17 & 6 & 0 & -2.887555 & -2.425028 & -0.000146 \\ 18 & 6 & 0 & -2.887598 & 2.425010 & -0.000010 \\ 19 & 6 & 0 & -3.578380 & 1.233545 & -0.000067 \\ 20 & 6 & 0 & -3.578389 & -1.233564 & -0.000127 \\ 21 & 1 & 0 & 0.977436 & -3.389279 & 0.000271 \\ 22 & 1 & 0 & 0.977444 & 3.389313 & -0.000114 \\ 23 & 1 & 0 & 4.664807 & 1.220328 & 0.000016 \\ 24 & 1 & 0 & 4.664862 & -1.220229 & 0.000176 \\ 25 & 1 & 0 & -0.977345 & -3.389366 & -0.000092 \\ 26 & 1 & 0 & 3.423180 & -3.369895 & 0.000316 \\ 27 & 1 & 0 & -0.977407 & 3.389377 & 0.000052 \\ 28 & 1 & 0 & 3.423155 & 3.369898 & -0.000101 \\ 29 & 1 & 0 & -3.423113 & -3.369883 & -0.000187 \\ 30 & 1 & 0 & -3.423167 & 3.369870 & 0.000018 \\ 31 & 1 & 0 & -4.664837 & 1.220239 & -0.000091 \\ 32 & 1 & 0 & -4.664884 & -1.220327 & -0.000155 \\ & & & & & \\ & 6 & & \end{array}$

25. Pyrene

\begin{tabular}{|c|c|c|c|c|c|}
\hline \multirow{2}{*}{$\begin{array}{l}\text { Center } \\
\text { Number }\end{array}$} & \multirow{2}{*}{$\begin{array}{l}\text { Atomic } \\
\text { Number }\end{array}$} & \multirow{2}{*}{\multicolumn{2}{|c|}{$\begin{array}{l}\text { Atomic } \\
\text { Type }\end{array}$}} & \multicolumn{2}{|c|}{ Coordinates (Angstroms } \\
\hline & & & & $\begin{array}{ll}\mathrm{X} & \mathrm{Y}\end{array}$ & $\mathrm{Z}$ \\
\hline 1 & 6 & 0 & -0.713986 & -0.000016 & 0.000033 \\
\hline 2 & 6 & 0 & 0.713972 & 0.000009 & -0.000020 \\
\hline 3 & 6 & 0 & -1.430238 & 1.237052 & 0.000108 \\
\hline 4 & 6 & 0 & -1.430237 & -1.237072 & 0.000004 \\
\hline 5 & 6 & 0 & 1.430243 & -1.237043 & -0.000117 \\
\hline 6 & 6 & 0 & 1.430239 & 1.237081 & -0.000026 \\
\hline 7 & 6 & 0 & -0.681643 & 2.465518 & 0.000115 \\
\hline 8 & 6 & 0 & -2.835595 & 1.211774 & 0.000169 \\
\hline 9 & 6 & 0 & -0.681607 & -2.465528 & -0.000032 \\
\hline 10 & 6 & 0 & -2.835588 & -1.211781 & 0.000130 \\
\hline 11 & 6 & 0 & 0.681656 & -2.465531 & -0.000117 \\
\hline 12 & 6 & 0 & 2.835594 & -1.211784 & -0.000183 \\
\hline 13 & 6 & 0 & 0.681628 & 2.465539 & 0.000027 \\
\hline 14 & 6 & 0 & 2.835583 & 1.211798 & -0.000095 \\
\hline 15 & 6 & 0 & -3.527300 & -0.000011 & 0.000189 \\
\hline 16 & 6 & 0 & 3.527294 & 0.000006 & -0.000201 \\
\hline 17 & 1 & 0 & -1.229348 & 3.404463 & 0.000173 \\
\hline 18 & 1 & 0 & -3.382460 & 2.151099 & 0.000245 \\
\hline
\end{tabular}




$\begin{array}{rrrrrr}19 & 1 & 0 & -1.229314 & -3.404468 & -0.000006 \\ 20 & 1 & 0 & -3.382437 & -2.151108 & 0.000153 \\ 21 & 1 & 0 & 1.229341 & -3.404489 & -0.000169 \\ 22 & 1 & 0 & 3.382408 & -2.151133 & -0.000246 \\ 23 & 1 & 0 & 1.229282 & 3.404517 & 0.000048 \\ 24 & 1 & 0 & 3.382434 & 2.151100 & -0.000114 \\ 25 & 1 & 0 & -4.613517 & -0.000027 & 0.000267 \\ 26 & 1 & 0 & 4.613520 & -0.000009 & -0.000268\end{array}$

26. Tetracene

\begin{tabular}{|c|c|c|c|c|c|}
\hline \multirow{2}{*}{$\begin{array}{l}\text { Center } \\
\text { Number }\end{array}$} & \multirow{2}{*}{$\begin{array}{l}\text { Atomic } \\
\text { Number }\end{array}$} & \multirow{2}{*}{\multicolumn{2}{|c|}{$\begin{array}{l}\text { Atomic } \\
\text { Type }\end{array}$}} & \multicolumn{2}{|c|}{ Coordinates (Angstroms } \\
\hline & & & & X $\quad Y$ & Z \\
\hline 1 & 6 & 0 & -0.000005 & 0.726508 & -0.000021 \\
\hline 2 & 6 & 0 & -0.000008 & -0.726428 & 0.000020 \\
\hline 3 & 6 & 0 & 1.236201 & 1.407776 & -0.000078 \\
\hline 4 & 6 & 0 & -1.236210 & 1.407775 & 0.000002 \\
\hline 5 & 6 & 0 & -1.236178 & -1.407754 & 0.000079 \\
\hline 6 & 6 & 0 & 1.236177 & -1.407723 & -0.000001 \\
\hline 7 & 6 & 0 & 2.452291 & 0.726215 & -0.000100 \\
\hline 8 & 6 & 0 & -2.452280 & 0.726191 & 0.000061 \\
\hline 9 & 6 & 0 & -2.452264 & -0.726214 & 0.000100 \\
\hline 10 & 6 & 0 & 2.452268 & -0.726189 & -0.000060 \\
\hline 11 & 6 & 0 & 3.713860 & 1.410492 & -0.000163 \\
\hline 12 & 6 & 0 & -3.713836 & 1.410485 & 0.000085 \\
\hline 13 & 6 & 0 & -3.713819 & -1.410503 & 0.000161 \\
\hline 14 & 6 & 0 & 3.713797 & -1.410506 & -0.000087 \\
\hline 15 & 6 & 0 & 4.893402 & 0.715891 & -0.000178 \\
\hline 16 & 6 & 0 & -4.893388 & 0.715913 & 0.000145 \\
\hline 17 & 6 & 0 & -4.893383 & -0.715946 & 0.000180 \\
\hline 18 & 6 & 0 & 4.893378 & -0.715971 & -0.000146 \\
\hline 19 & 1 & 0 & 1.236438 & 2.495536 & -0.000107 \\
\hline 20 & 1 & 0 & -1.236453 & 2.495538 & -0.000027 \\
\hline 21 & 1 & 0 & -1.236384 & -2.495515 & 0.000108 \\
\hline 22 & 1 & 0 & 1.236393 & -2.495481 & 0.000027 \\
\hline 23 & 1 & 0 & 3.713103 & 2.497483 & -0.000191 \\
\hline 24 & 1 & 0 & -3.713074 & 2.497474 & 0.000055 \\
\hline 25 & 1 & 0 & -3.713069 & -2.497491 & 0.000192 \\
\hline 26 & 1 & 0 & 3.713026 & -2.497489 & -0.000056 \\
\hline 27 & 1 & 0 & 5.839899 & 1.248572 & -0.000222 \\
\hline 28 & 1 & 0 & -5.839878 & 1.248600 & 0.000163 \\
\hline 29 & 1 & 0 & -5.839859 & -1.248631 & 0.000228 \\
\hline 30 & 1 & 0 & 5.839839 & -1.248665 & -0.000160 \\
\hline
\end{tabular}

27. Triphenylene

\begin{tabular}{lllrrr} 
Center & \multicolumn{2}{c}{ Atomic } & Atomic & \multicolumn{2}{c}{ Coordinates (Angstroms) } \\
Number & Number & Type & X & Y & Z \\
- \hdashline 1 & 6 & 0 & 1.133022 & -0.897066 & -0.006577 \\
2 & 6 & 0 & -0.237112 & -1.425359 & -0.005313 \\
3 & 6 & 0 & 1.353076 & 0.507194 & 0.002342 \\
4 & 6 & 0 & 2.257469 & -1.755987 & -0.010297
\end{tabular}




$\begin{array}{cccccc}5 & 6 & 0 & -1.343089 & -0.532560 & 0.003643 \\ 6 & 6 & 0 & -0.493634 & -2.816806 & -0.007018 \\ 7 & 6 & 0 & 0.210506 & 1.429401 & -0.000587 \\ 8 & 6 & 0 & 2.686217 & 0.981146 & 0.012830 \\ 9 & 6 & 0 & 3.552705 & -1.266518 & -0.003279 \\ 10 & 6 & 0 & -1.115679 & 0.917931 & 0.000747 \\ 11 & 6 & 0 & -2.649353 & -1.076254 & 0.010305 \\ 12 & 6 & 0 & -1.782196 & -3.323693 & -0.000822 \\ 13 & 6 & 0 & 0.392231 & 2.832514 & -0.005537 \\ 14 & 6 & 0 & 3.769751 & 0.119091 & 0.009998 \\ 15 & 6 & 0 & -2.192863 & 1.835321 & -0.000072 \\ 16 & 6 & 0 & -2.873498 & -2.442645 & 0.007785 \\ 17 & 6 & 0 & -0.679422 & 3.709450 & -0.006752 \\ 18 & 6 & 0 & -1.988053 & 3.204729 & -0.003160 \\ 19 & 1 & 0 & 2.117083 & -2.829622 & -0.018600 \\ 20 & 1 & 0 & 0.331284 & -3.518092 & -0.012553 \\ 21 & 1 & 0 & 2.880697 & 2.046280 & 0.025259 \\ 22 & 1 & 0 & 4.393238 & -1.954320 & -0.007067 \\ 23 & 1 & 0 & -3.508313 & -0.417176 & 0.017624 \\ 24 & 1 & 0 & -1.943292 & -4.397775 & -0.002861 \\ 25 & 1 & 0 & 1.392281 & 3.247624 & -0.010525 \\ 26 & 1 & 0 & 4.780295 & 0.516973 & 0.018866 \\ 27 & 1 & 0 & -3.212456 & 1.470876 & 0.001538 \\ 28 & 1 & 0 & -3.889465 & -2.826513 & 0.013819 \\ 29 & 1 & 0 & -0.504039 & 4.781272 & -0.011222 \\ 30 & 1 & 0 & -2.837790 & 3.881145 & -0.003684 \\ -----------------------------\end{array}$

III. Cartesian Coordinates of the Watson-Crick Base Pairs Optimized at B3LYP/6-31++G(d,p) Level 1. Guanine:Cytosine $(\mathrm{G} \cdot \mathrm{C})$

\begin{tabular}{|c|c|c|c|c|}
\hline \multirow{2}{*}{$\begin{array}{l}\text { Center } \\
\text { Number }\end{array}$} & \multirow{2}{*}{$\begin{array}{l}\text { Atomic } \\
\text { Number }\end{array}$} & \multirow{2}{*}{$\begin{array}{l}\text { Atomic } \\
\text { Type }\end{array}$} & \multicolumn{2}{|c|}{ Coordinates (Angstroms) } \\
\hline & & & $X \quad Y$ & Z \\
\hline 1 & 7 & 4.678761 & 0.477325 & -0.005662 \\
\hline 2 & 6 & 4.976616 & -0.878433 & -0.005670 \\
\hline 3 & 1 & 5.997819 & -1.233798 & -0.008429 \\
\hline 4 & 7 & 3.908643 & -1.632287 & -0.002255 \\
\hline 5 & 6 & 2.850178 & -0.738404 & 0.000319 \\
\hline 6 & 6 & 1.436110 & -0.951945 & 0.004075 \\
\hline 7 & 8 & 0.808342 & -2.024569 & 0.006055 \\
\hline 8 & 7 & 0.730962 & 0.267524 & 0.005575 \\
\hline 9 & 1 & -0.298981 & 0.180278 & 0.008370 \\
\hline 10 & 6 & 1.292880 & 1.523359 & 0.003444 \\
\hline 11 & 7 & 0.440261 & 2.571685 & 0.006388 \\
\hline 12 & 1 & -0.576431 & 2.462518 & 0.003615 \\
\hline 13 & 1 & 0.848364 & 3.492294 & 0.001053 \\
\hline 14 & 7 & 2.602605 & 1.732395 & -0.000482 \\
\hline 15 & 6 & 3.310966 & 0.584050 & -0.001734 \\
\hline 16 & 7 & -4.344966 & 0.916327 & -0.003873 \\
\hline 17 & 6 & -4.952775 & -0.302831 & -0.004745 \\
\hline 18 & 1 & -6.037164 & -0.305224 & -0.007062 \\
\hline 19 & 6 & -4.208153 & -1.438023 & -0.002797 \\
\hline
\end{tabular}




$\begin{array}{rrrrrr}20 & 1 & 0 & -4.673363 & -2.415404 & -0.003583 \\ 21 & 6 & 0 & -2.771920 & -1.281492 & 0.000287 \\ 22 & 7 & 0 & -1.969191 & -2.351693 & 0.002486 \\ 23 & 1 & 0 & -2.361598 & -3.279792 & 0.001435 \\ 24 & 1 & 0 & -0.938006 & -2.238188 & 0.003188 \\ 25 & 7 & 0 & -2.196224 & -0.071074 & 0.001111 \\ 26 & 6 & 0 & -2.943584 & 1.062737 & -0.001227 \\ 27 & 8 & 0 & -2.471733 & 2.207705 & -0.001138 \\ 28 & 1 & 0 & 5.326967 & 1.251188 & -0.007147 \\ 29 & 1 & 0 & -4.878349 & 1.775530 & -0.005502\end{array}$

2. 9-MethylGuanine:1-MethylCytosine (9-MetG·1-MetC)

\begin{tabular}{|c|c|c|c|c|c|}
\hline \multirow{2}{*}{$\begin{array}{l}\text { Center } \\
\text { Number }\end{array}$} & \multirow{2}{*}{$\begin{array}{l}\text { Atomic } \\
\text { Number }\end{array}$} & \multirow{2}{*}{\multicolumn{2}{|c|}{$\begin{array}{l}\text { Atomic } \\
\text { Type }\end{array}$}} & \multicolumn{2}{|c|}{ Coordinates (Angstroms } \\
\hline & & & & $\begin{array}{ll}X & Y\end{array}$ & $\mathrm{Z}$ \\
\hline 1 & 7 & 0 & 4.653639 & -0.334745 & 0.004181 \\
\hline 2 & 6 & 0 & 4.962717 & 1.018984 & 0.005077 \\
\hline 3 & 1 & 0 & 5.991542 & 1.354588 & 0.007895 \\
\hline 4 & 7 & 0 & 3.909775 & 1.795951 & 0.002337 \\
\hline 5 & 6 & 0 & 2.838934 & 0.920441 & -0.000669 \\
\hline 6 & 6 & 0 & 1.428526 & 1.153710 & -0.004053 \\
\hline 7 & 8 & 0 & 0.815007 & 2.234589 & -0.005265 \\
\hline 8 & 7 & 0 & 0.705660 & -0.056643 & -0.006242 \\
\hline 9 & 1 & 0 & -0.323329 & 0.043126 & -0.008491 \\
\hline 10 & 6 & 0 & 1.250616 & -1.319775 & -0.005087 \\
\hline 11 & 7 & 0 & 0.382630 & -2.356464 & -0.008715 \\
\hline 12 & 1 & 0 & -0.632245 & -2.231461 & -0.005781 \\
\hline 13 & 1 & 0 & 0.777715 & -3.282563 & -0.003814 \\
\hline 14 & 7 & 0 & 2.556869 & -1.547548 & -0.001549 \\
\hline 15 & 6 & 0 & 3.283033 & -0.408199 & 0.000399 \\
\hline 16 & 7 & 0 & -4.397859 & -0.665331 & 0.003165 \\
\hline 17 & 6 & 0 & -4.963177 & 0.574537 & 0.004408 \\
\hline 18 & 1 & 0 & -6.047624 & 0.604791 & 0.006702 \\
\hline 19 & 6 & 0 & -4.207360 & 1.704371 & 0.002885 \\
\hline 20 & 1 & 0 & -4.668256 & 2.684015 & 0.003962 \\
\hline 21 & 6 & 0 & -2.777967 & 1.536661 & -0.000129 \\
\hline 22 & 7 & 0 & -1.958567 & 2.596395 & -0.001692 \\
\hline 23 & 1 & 0 & -2.339115 & 3.529203 & -0.000134 \\
\hline 24 & 1 & 0 & -0.929631 & 2.470098 & -0.002536 \\
\hline 25 & 7 & 0 & -2.225282 & 0.317042 & -0.001512 \\
\hline 26 & 6 & 0 & -2.988250 & -0.805835 & 0.000137 \\
\hline 27 & 8 & 0 & -2.516870 & -1.953598 & -0.000814 \\
\hline 28 & 6 & 0 & 5.572378 & -1.460250 & 0.006227 \\
\hline 29 & 1 & 0 & 5.416123 & -2.080049 & 0.893683 \\
\hline 30 & 1 & 0 & 5.421518 & -2.078935 & -0.882945 \\
\hline 31 & 1 & 0 & 6.595157 & -1.077915 & 0.009578 \\
\hline 32 & 6 & 0 & -5.203488 & -1.887631 & 0.004915 \\
\hline 33 & 1 & 0 & -4.977281 & -2.486809 & -0.880012 \\
\hline 34 & 1 & 0 & -4.973908 & -2.486552 & 0.889155 \\
\hline 35 & 1 & 0 & -6.259603 & -1.612167 & 0.006894 \\
\hline
\end{tabular}

3. Adenine:Thymine $(\mathrm{A} \cdot \mathrm{T})$ 


\begin{tabular}{|c|c|c|c|c|c|}
\hline \multirow{2}{*}{$\begin{array}{l}\text { Center } \\
\text { Number }\end{array}$} & \multirow{2}{*}{$\begin{array}{l}\text { Atomic } \\
\text { Number }\end{array}$} & \multirow{2}{*}{\multicolumn{2}{|c|}{$\begin{array}{r}\text { Atomic } \\
\text { Type }\end{array}$}} & \multicolumn{2}{|c|}{ Coordinates (Angstroms } \\
\hline & & & & $\begin{array}{ll}X & Y\end{array}$ & Z \\
\hline 1 & 7 & 0 & -5.002327 & 0.542309 & 0.001509 \\
\hline 2 & 6 & 0 & -5.247206 & -0.817228 & 0.000561 \\
\hline 3 & 1 & 0 & -6.254092 & -1.211755 & 0.000887 \\
\hline 4 & 7 & 0 & -4.151681 & -1.539314 & -0.000457 \\
\hline 5 & 6 & 0 & -3.129976 & -0.604268 & -0.000390 \\
\hline 6 & 6 & 0 & -1.721338 & -0.735703 & -0.001103 \\
\hline 7 & 7 & 0 & -1.095063 & -1.925289 & -0.002323 \\
\hline 8 & 1 & 0 & -1.647982 & -2.768022 & -0.001737 \\
\hline 9 & 1 & 0 & -0.074461 & -1.976738 & -0.001434 \\
\hline 10 & 7 & 0 & -0.989267 & 0.403719 & -0.000741 \\
\hline 11 & 6 & 0 & -1.614435 & 1.598747 & 0.000269 \\
\hline 12 & 1 & 0 & -0.954183 & 2.462378 & 0.000415 \\
\hline 13 & 7 & 0 & -2.925803 & 1.839802 & 0.001096 \\
\hline 14 & 6 & 0 & -3.633986 & 0.699664 & 0.000744 \\
\hline 15 & 7 & 0 & 3.892466 & 1.575093 & -0.000160 \\
\hline 16 & 6 & 0 & 4.594852 & 0.390069 & 0.000479 \\
\hline 17 & 1 & 0 & 5.675202 & 0.491552 & 0.000871 \\
\hline 18 & 6 & 0 & 3.980104 & -0.817608 & 0.000644 \\
\hline 19 & 6 & 0 & 4.713026 & -2.128142 & 0.001522 \\
\hline 20 & 1 & 0 & 4.446 & -2.725023 & 0.880125 \\
\hline 21 & 1 & 0 & 4.446933 & -2.726118 & -0.876498 \\
\hline 22 & 1 & 0 & 5.795650 & -1.971897 & 0.001754 \\
\hline 23 & 6 & 0 & 2.514923 & -0.831880 & 0.000149 \\
\hline 24 & 8 & 0 & 1.844663 & -1.872222 & 0.000200 \\
\hline 25 & 7 & 0 & 1.887749 & 0.409656 & -0.000404 \\
\hline 26 & 1 & 0 & 0.840362 & 0.417203 & -0.000950 \\
\hline 27 & 6 & 0 & 2.502658 & 1.645417 & -0.000640 \\
\hline 28 & 8 & 0 & 1.902 & 2.711962 & -0.001165 \\
\hline 29 & 1 & 0 & 4.369302 & 2.465857 & -0.000285 \\
\hline 30 & 1 & 0 & -5.682726 & 1.288391 & 0.001526 \\
\hline
\end{tabular}

4. 9-MethylAdenine:1-MethylThymine (9-MetA·1-MetT)

\begin{tabular}{|c|c|c|c|c|}
\hline \multirow{2}{*}{$\begin{array}{l}\text { Center } \\
\text { Number }\end{array}$} & \multirow{2}{*}{$\begin{array}{l}\text { Atomic } \\
\text { Number }\end{array}$} & \multirow{2}{*}{$\begin{array}{l}\text { Atomic } \\
\text { Type }\end{array}$} & \multicolumn{2}{|c|}{ Coordinates (Angstroms) } \\
\hline & & & $\begin{array}{ll}X & Y\end{array}$ & Z \\
\hline 1 & 7 & 4.922382 & -0.393934 & 0.000445 \\
\hline 2 & 6 & 5.186971 & 0.961983 & 0.000443 \\
\hline 3 & 1 & 6.204505 & 1.330830 & 0.000714 \\
\hline 4 & 7 & 4.110058 & 1.714812 & 0.000040 \\
\hline 5 & 6 & 3.069572 & 0.804110 & -0.000217 \\
\hline 6 & 6 & 1.663985 & 0.962591 & -0.000662 \\
\hline 7 & 7 & 1.060084 & 2.163998 & -0.001101 \\
\hline 8 & 1 & 1.629833 & 2.995421 & -0.000517 \\
\hline 9 & 1 & 0.040344 & 2.235501 & -0.000757 \\
\hline 10 & 7 & 0.909583 & -0.162719 & -0.000753 \\
\hline 11 & 6 & 1.510984 & -1.369860 & -0.000462 \\
\hline 12 & 1 & 0.833004 & -2.219693 & -0.000577 \\
\hline 13 & 7 & 2.817553 & -1.636414 & -0.000054 \\
\hline
\end{tabular}




$\begin{array}{rrrrrr}14 & 6 & 0 & 3.549134 & -0.509441 & 0.000053 \\ 15 & 7 & 0 & -3.999528 & -1.267191 & 0.000154 \\ 16 & 6 & 0 & -4.656893 & -0.055651 & 0.000579 \\ 17 & 1 & 0 & -5.739996 & -0.124790 & 0.001029 \\ 18 & 6 & 0 & -4.025794 & 1.145829 & 0.000472 \\ 19 & 6 & 0 & -4.748378 & 2.462677 & 0.000950 \\ 20 & 1 & 0 & -4.476894 & 3.058167 & 0.879172 \\ 21 & 1 & 0 & -4.477553 & 3.058441 & -0.877289 \\ 22 & 1 & 0 & -5.832456 & 2.315968 & 0.001335 \\ 23 & 6 & 0 & -2.565817 & 1.143610 & -0.000065 \\ 24 & 8 & 0 & -1.875659 & 2.172491 & -0.000113 \\ 25 & 7 & 0 & -1.967919 & -0.109793 & -0.000516 \\ 26 & 1 & 0 & -0.921054 & -0.141852 & -0.000931 \\ 27 & 6 & 0 & -2.604659 & -1.333824 & -0.000540 \\ 28 & 8 & 0 & -2.007851 & -2.405282 & -0.000709 \\ 29 & 6 & 0 & 5.876537 & -1.491818 & 0.001088 \\ 30 & 1 & 0 & 5.739196 & -2.112679 & 0.890116 \\ 31 & 1 & 0 & 5.738038 & -2.114552 & -0.886433 \\ 32 & 1 & 0 & 6.886943 & -1.079096 & -0.000008 \\ 33 & 6 & 0 & -4.723162 & -2.540127 & 0.000359 \\ 34 & 1 & 0 & -4.461633 & -3.124362 & -0.885011 \\ 35 & 1 & 0 & -4.460797 & -3.124563 & 0.885349 \\ 36 & 1 & 0 & -5.793773 & -2.332199 & 0.000891 \\ --------------------------------------------------------\end{array}$

IV. Cartesian Coordinates of the Nucleosides and Nucleosides' Analogs Optimized at B3LYP/6$31++G(d, p)$ Level

1. anti-dAdo

\begin{tabular}{|c|c|c|c|c|c|}
\hline \multirow{2}{*}{$\begin{array}{l}\text { Center } \\
\text { Number }\end{array}$} & Atomic & \multicolumn{2}{|c|}{ Atomic } & \multicolumn{2}{|c|}{ Coordinates (Angstroms } \\
\hline & Numbe & & Type & X $\quad Y$ & Z \\
\hline 1 & 6 & 0 & -1.545119 & -0.486435 & 0.053143 \\
\hline 2 & 7 & 0 & -0.269275 & 0.029740 & -0.082015 \\
\hline 3 & 6 & 0 & -0.427710 & 1.389692 & -0.295353 \\
\hline 4 & 7 & 0 & -1.682417 & 1.776207 & -0.318649 \\
\hline 5 & 6 & 0 & -2.399282 & 0.610156 & -0.104022 \\
\hline 6 & 7 & 0 & -1.910702 & -1.756615 & 0.272099 \\
\hline 7 & 6 & 0 & -3.240524 & -1.882914 & 0.333133 \\
\hline 8 & 7 & 0 & -4.178581 & -0.929239 & 0.200885 \\
\hline 9 & 6 & 0 & -3.779835 & 0.337637 & -0.018320 \\
\hline 10 & 6 & 0 & 0.974551 & -0.705342 & 0.050385 \\
\hline 11 & 8 & 0 & 1.740772 & -0.130319 & 1.115448 \\
\hline 12 & 6 & 0 & 3.127639 & -0.039506 & 0.765798 \\
\hline 13 & 6 & 0 & & -0.7 & -0.599756 \\
\hline 14 & 6 & 0 & 1.880635 & -0.654489 & -1.190134 \\
\hline 15 & 6 & 0 & 3.583367 & 1.415404 & 0.788106 \\
\hline 16 & 8 & 0 & 2.973679 & 2.124958 & -0.294021 \\
\hline 17 & 8 & 0 & 3.583680 & -2.143466 & -0.445932 \\
\hline 18 & 7 & 0 & -4.721032 & 1.308886 & -0.131870 \\
\hline 19 & 1 & 0 & 4.680485 & 1.443406 & 0.700469 \\
\hline 20 & 1 & 0 & 3.297169 & 1.853513 & 1.753062 \\
\hline 21 & 1 & 0 & 3.705761 & -0.574286 & 1.533046 \\
\hline
\end{tabular}




$\begin{array}{rrrrrr}22 & 1 & 0 & 0.689545 & -1.728850 & 0.309315 \\ 23 & 1 & 0 & 4.035320 & -0.264365 & -1.230011 \\ 24 & 1 & 0 & 1.762969 & 0.302272 & -1.703271 \\ 25 & 1 & 0 & 1.675739 & -1.469683 & -1.887022 \\ 26 & 1 & 0 & 0.434110 & 2.033404 & -0.404649 \\ 27 & 1 & 0 & -4.449205 & 2.241717 & -0.398470 \\ 28 & 1 & 0 & -5.691961 & 1.039965 & -0.161359 \\ 29 & 1 & 0 & -3.617810 & -2.886856 & 0.510483 \\ 30 & 1 & 0 & 4.465288 & -2.243787 & -0.063497 \\ 31 & 1 & 0 & 3.223129 & 3.055834 & -0.243087 \\ -\end{array}$

\section{2. syn-dAdo}

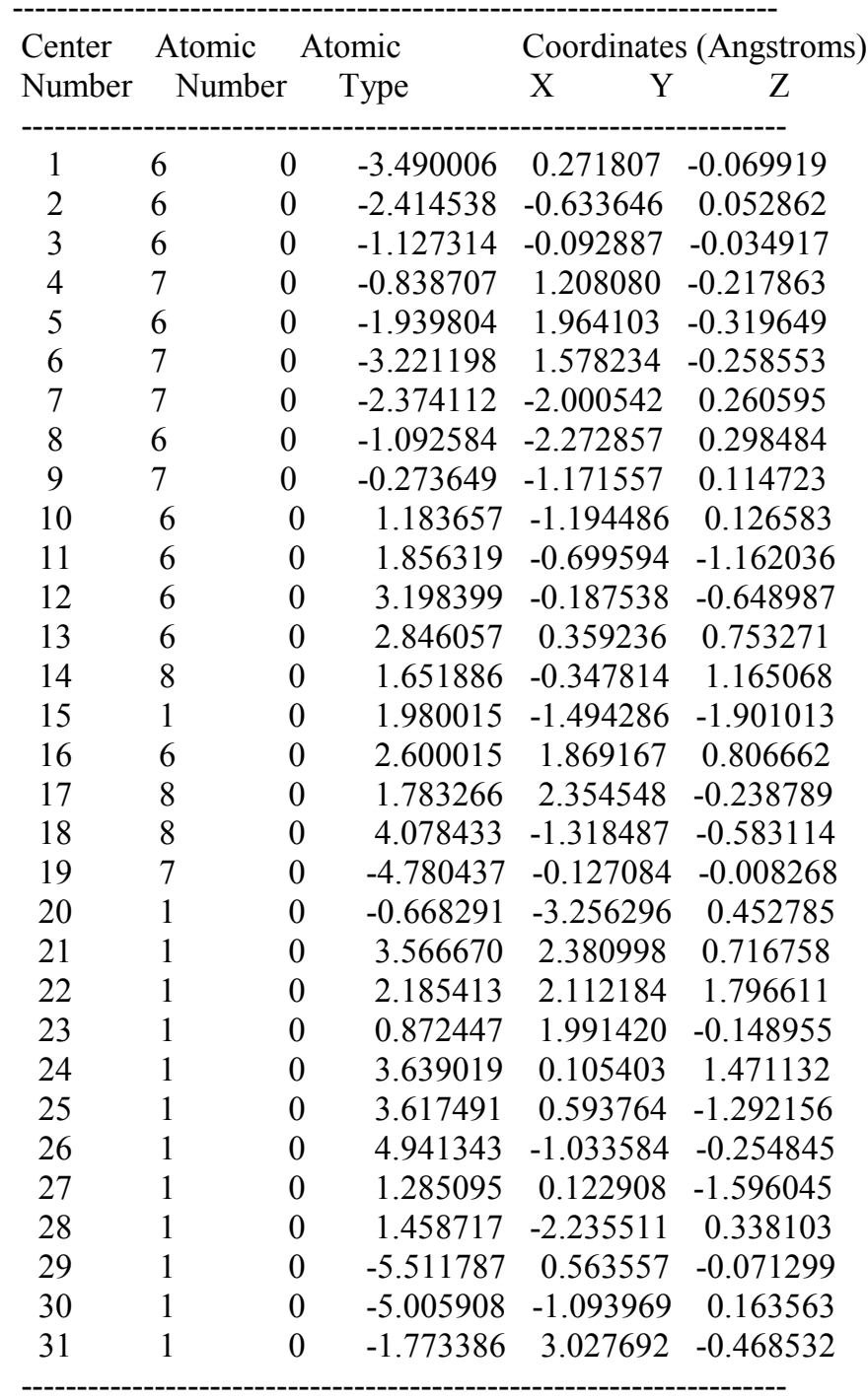

\section{3. anti-dGuo}

\begin{tabular}{lcccc} 
Center & Atomic & Atomic & & \multicolumn{2}{c}{ Coordinates (Angstroms) } \\
Number & Number & Type & X & Y
\end{tabular}




\begin{tabular}{|c|c|c|c|c|c|}
\hline 1 & 6 & 0 & -2.049474 & 0.586241 & -1.238875 \\
\hline 2 & 6 & 0 & -1.102268 & 0.615411 & -0.029083 \\
\hline 3 & 8 & 0 & -1.897662 & 0.228168 & 1.097684 \\
\hline 4 & 6 & 0 & -3.296144 & 0.280520 & 0.786622 \\
\hline 5 & 6 & 0 & -3.404887 & 0.912161 & -0.622833 \\
\hline 6 & 6 & 0 & -3.922918 & -1.103257 & 0.921645 \\
\hline 7 & 8 & 0 & -3.456260 & -1.946486 & -0.134556 \\
\hline 8 & 7 & 0 & 0.034937 & -0.278943 & -0.135008 \\
\hline 9 & 6 & 0 & 0.016731 & -1.661033 & -0.287752 \\
\hline 10 & 7 & 0 & 1.212798 & -2.192698 & -0.301268 \\
\hline 11 & 6 & 0 & 2.073749 & -1.122630 & -0.146925 \\
\hline 12 & 6 & 0 & 1.361106 & 0.071751 & -0.032125 \\
\hline 13 & 7 & 0 & 1.838442 & 1.335596 & 0.119937 \\
\hline 14 & 6 & 0 & 3.146901 & 1.383317 & 0.193883 \\
\hline 15 & 7 & 0 & 3.949145 & 0.274716 & 0.104067 \\
\hline 16 & 6 & 0 & 3.509692 & -1.082364 & -0.078420 \\
\hline 17 & 8 & 0 & 4.346200 & -1.971747 & -0.141165 \\
\hline 18 & 7 & 0 & 3.771628 & 2.590331 & 0.424008 \\
\hline 19 & 8 & 0 & -3.513886 & 2.338315 & -0.562308 \\
\hline 20 & 1 & 0 & -5.018413 & -1.000276 & 0.879353 \\
\hline 21 & 1 & 0 & -3.646730 & -1.512901 & 1.901757 \\
\hline 22 & 1 & 0 & -3.781847 & 0.932704 & 1.526492 \\
\hline 23 & 1 & 0 & -0.686104 & 1.612029 & 0.140484 \\
\hline 24 & 1 & 0 & -0.919327 & -2.196229 & -0.359064 \\
\hline 25 & 1 & 0 & 4.951266 & 0.360865 & 0.228073 \\
\hline 26 & 1 & 0 & 4.693649 & 2.728785 & 0.034088 \\
\hline 27 & 1 & 0 & 3.154573 & 3.385692 & 0.329074 \\
\hline 28 & 1 & 0 & -4.236956 & 0.486849 & -1.196465 \\
\hline 29 & 1 & 0 & -2.073757 & -0.414350 & -1.674824 \\
\hline 30 & 1 & 0 & -1.767121 & 1.311259 & -2.005048 \\
\hline 31 & 1 & 0 & -3.776928 & -2.845176 & 0.010365 \\
\hline 32 & 1 & 0 & -4.363007 & 2.581031 & -0.170489 \\
\hline
\end{tabular}

\section{4. syn-dGuo}

\begin{tabular}{|c|c|c|c|c|}
\hline \multirow{2}{*}{$\begin{array}{l}\text { Center } \\
\text { Number }\end{array}$} & \multirow{2}{*}{$\begin{array}{l}\text { Atomic } \\
\text { Number }\end{array}$} & \multirow{2}{*}{$\begin{array}{l}\text { Atomic } \\
\text { Type }\end{array}$} & \multicolumn{2}{|c|}{ Coordinates (Angstroms } \\
\hline & & & $\mathrm{X}$ & Z \\
\hline 1 & 6 & 1.961341 & -0.717471 & -1.179066 \\
\hline 2 & 6 & 1.360958 & -1.296010 & 0.111283 \\
\hline 3 & 8 & 1.765952 & -0.417527 & 1.154526 \\
\hline 4 & 6 & 2.911594 & 0.365868 & 0.750531 \\
\hline 5 & 6 & 3.265030 & -0.098028 & -0.683945 \\
\hline 6 & 7 & -0.089441 & -1.417489 & 0.123383 \\
\hline 7 & 6 & -1.042013 & -0.430394 & 0.008903 \\
\hline 8 & 6 & -2.271145 & -1.087069 & 0.065077 \\
\hline 9 & 7 & -2.095530 & -2.447273 & 0.221337 \\
\hline 10 & 6 & -0.798724 & -2.605554 & 0.257973 \\
\hline 11 & 7 & -0.812454 & 0.905064 & -0.131091 \\
\hline 12 & 6 & -1.908674 & 1.630219 & -0.202761 \\
\hline 13 & 7 & -3.162707 & 1.083751 & -0.164174 \\
\hline 14 & 6 & -3.482293 & -0.312981 & -0.023239 \\
\hline
\end{tabular}




\begin{tabular}{|c|c|c|c|c|c|}
\hline 15 & 8 & 0 & -4.654799 & -0.649551 & 0.007193 \\
\hline 16 & 7 & 0 & -1.809754 & 2.994971 & -0.268078 \\
\hline 17 & 6 & 0 & 2.576548 & 1.852155 & 0.886086 \\
\hline 18 & 8 & 0 & 1.627878 & 2.302811 & -0.064696 \\
\hline 19 & 8 & 0 & 4.248886 & -1.140282 & -0.685435 \\
\hline 20 & 1 & 0 & 2.139440 & -1.482672 & -1.937617 \\
\hline 21 & 1 & 0 & -0.282078 & -3.548594 & 0.376428 \\
\hline 22 & 1 & 0 & -2.557109 & 3.524034 & -0.692240 \\
\hline 23 & 1 & 0 & -0.876286 & 3.358189 & -0.413525 \\
\hline 24 & 1 & 0 & 3.490123 & 2.438929 & 0.730906 \\
\hline 25 & 1 & 0 & 2.231880 & 2.033353 & 1.915112 \\
\hline 26 & 1 & 0 & 0.811120 & 1.757912 & 0.015783 \\
\hline 27 & 1 & 0 & 3.737947 & 0.127617 & 1.435546 \\
\hline 28 & 1 & 0 & 3.589795 & 0.741511 & -1.308786 \\
\hline 29 & 1 & 0 & 5.093863 & -0.785813 & -0.378813 \\
\hline 30 & 1 & 0 & 1.307293 & 0.056066 & -1.585780 \\
\hline 31 & 1 & 0 & 1.743177 & -2.306329 & 0.302031 \\
\hline 32 & 1 & 0 & -3.979008 & 1.684609 & -0.166435 \\
\hline
\end{tabular}

5. anit-dCyd

\begin{tabular}{|c|c|c|c|c|}
\hline \multirow{2}{*}{$\begin{array}{l}\text { Center } \\
\text { Number }\end{array}$} & \multirow{2}{*}{$\begin{array}{l}\text { Atomic } \\
\text { Number }\end{array}$} & \multirow{2}{*}{$\begin{array}{r}\text { Atomic } \\
\text { Type }\end{array}$} & \multicolumn{2}{|c|}{ Coordinates (Angstroms } \\
\hline & & & $\begin{array}{ll}X & Y\end{array}$ & Z \\
\hline 1 & 8 & 1.355183 & 0.023191 & -1.310061 \\
\hline 2 & 6 & 0.484584 & -0.970501 & -0.787604 \\
\hline 3 & 6 & 1.230540 & -1.631721 & 0.383595 \\
\hline 4 & 6 & 2.246589 & -0.560691 & 0.800742 \\
\hline 5 & 6 & 2.569739 & 0.103658 & -0.543284 \\
\hline 6 & 7 & -0.796777 & -0.335683 & -0.384624 \\
\hline 7 & 6 & -1.912605 & -1.221933 & -0.231030 \\
\hline 8 & 7 & -3.119059 & -0.668165 & 0.089983 \\
\hline 9 & 6 & -3.228820 & 0.637286 & 0.272465 \\
\hline 10 & 6 & -2.125620 & 1.543358 & 0.154571 \\
\hline 11 & 6 & -0.920762 & 1.001729 & -0.186751 \\
\hline 12 & 8 & -1.716848 & -2.426405 & -0.388712 \\
\hline 13 & 7 & -4.473419 & 1.117191 & 0.560369 \\
\hline 14 & 8 & 3.460934 & -1.061893 & 1.353901 \\
\hline 15 & 6 & 3.055435 & 1.539799 & -0.489317 \\
\hline 16 & 8 & 2.11 & 2.336739 & 0.241611 \\
\hline 17 & 1 & 4.039028 & 1.543522 & 0.000014 \\
\hline 18 & 1 & 3.167194 & 1.915870 & -1.514895 \\
\hline 19 & 1 & 2.442589 & 3.244111 & 0.286635 \\
\hline 20 & 1 & 3.353176 & -0.491211 & -1.037196 \\
\hline 21 & 1 & 1.796398 & 0.175458 & 1.477542 \\
\hline 22 & 1 & 3.300717 & -1.352179 & 2.260634 \\
\hline 23 & 1 & 1.772952 & -2.511378 & 0.021183 \\
\hline 24 & 1 & 0.224303 & -1.688109 & -1.566492 \\
\hline 25 & 1 & -0.020457 & 1.591227 & -0.314859 \\
\hline 26 & 1 & -2.232823 & 2.610470 & 0.304875 \\
\hline 27 & 1 & -5.198868 & 0.440663 & 0.746853 \\
\hline 28 & 1 & -4.599642 & 2.060713 & 0.887960 \\
\hline 29 & 1 & 0.549041 & -1.951511 & 1.173417 \\
\hline
\end{tabular}


6. syn-dCyd

\begin{tabular}{|c|c|c|c|c|c|}
\hline \multirow{2}{*}{$\begin{array}{l}\text { Center } \\
\text { Number }\end{array}$} & \multirow{2}{*}{$\begin{array}{l}\text { Atomic } \\
\text { Number }\end{array}$} & \multirow{2}{*}{\multicolumn{2}{|c|}{$\begin{array}{l}\text { Atomic } \\
\text { Type }\end{array}$}} & \multicolumn{2}{|c|}{ Coordinates (Angstroms } \\
\hline & & & & X $\quad Y$ & $\mathrm{Z}$ \\
\hline 1 & 6 & 0 & -1.986365 & -1.525184 & 0.269024 \\
\hline 2 & 7 & 0 & -0.951201 & -0.676770 & 0.006049 \\
\hline 3 & 6 & 0 & -1.213241 & 0.713006 & -0.241493 \\
\hline 4 & 7 & 0 & -2.512937 & 1.116778 & -0.280033 \\
\hline 5 & 6 & 0 & -3.501436 & 0.269277 & -0.052054 \\
\hline 6 & 6 & 0 & -3.282022 & -1.115466 & 0.249273 \\
\hline 7 & 6 & 0 & 0.423735 & -1.203703 & 0.088910 \\
\hline 8 & 6 & 0 & 1.330473 & -0.970100 & -1.120722 \\
\hline 9 & 6 & 0 & 2.738586 & -0.936977 & -0.486822 \\
\hline 10 & 6 & 0 & 2.474360 & -0.398894 & 0.954978 \\
\hline 11 & 8 & 0 & 1.071324 & -0.638599 & 1.216070 \\
\hline 12 & 6 & 0 & 2.801402 & 1.081891 & 1.146411 \\
\hline 13 & 8 & 0 & 3.697362 & -0.206057 & -1.219925 \\
\hline 14 & 8 & 0 & -0.272145 & 1.487218 & -0.422560 \\
\hline 15 & 7 & 0 & -4.765684 & 0.758884 & -0.121086 \\
\hline 16 & 1 & 0 & 3.040867 & -0.972818 & 1.700452 \\
\hline 17 & 1 & 0 & 3.138938 & -1.954246 & -0.421195 \\
\hline 18 & 1 & 0 & 0.291795 & -2.282293 & 0.255805 \\
\hline 19 & 1 & 0 & -1.713896 & -2.551113 & 0.492342 \\
\hline 20 & 1 & 0 & -4.090891 & -1.805306 & 0.454589 \\
\hline 21 & 1 & 0 & -4.885117 & 1.748580 & -0.278076 \\
\hline 22 & 1 & 0 & -5.566183 & 0.197552 & 0.117090 \\
\hline 23 & 1 & 0 & 1.113099 & -0.010209 & -1.589790 \\
\hline 24 & 1 & 0 & 1.226303 & -1.763121 & -1.865980 \\
\hline 25 & 1 & 0 & 3.886226 & 1.192408 & 1.244607 \\
\hline 26 & 8 & 0 & 2.411695 & 1.897360 & 0.047902 \\
\hline 27 & 1 & 0 & 2.337984 & 1.422764 & 2.082509 \\
\hline 28 & 1 & 0 & 1.437414 & 1.855213 & -0.050355 \\
\hline 29 & 1 & 0 & 3.413363 & 0.727861 & -1.193428 \\
\hline
\end{tabular}

7. anti-dThd

\begin{tabular}{|c|c|c|c|c|}
\hline \multirow{2}{*}{$\begin{array}{l}\text { Center } \\
\text { Number }\end{array}$} & \multirow{2}{*}{$\begin{array}{l}\text { Atomic } \\
\text { Number }\end{array}$} & \multirow{2}{*}{$\begin{array}{l}\text { Atomic } \\
\text { Type }\end{array}$} & \multicolumn{2}{|c|}{ Coordinates (Angstroms } \\
\hline & & & $\begin{array}{ll}X & Y\end{array}$ & $\mathrm{Z}$ \\
\hline 1 & 6 & -1.623316 & -0.646473 & 1.176401 \\
\hline 2 & 6 & -0.831442 & -0.942641 & -0.105342 \\
\hline 3 & 8 & -1.425548 & -0.124923 & -1.119897 \\
\hline 4 & 6 & -2.740683 & 0.295839 & -0.732991 \\
\hline 5 & 6 & -3.023304 & -0.347136 & 0.650397 \\
\hline 6 & 6 & -2.831500 & 1.816299 & -0.765812 \\
\hline 7 & 8 & -2.031341 & 2.362701 & 0.287860 \\
\hline 8 & 7 & 0.594709 & -0.623264 & -0.034163 \\
\hline 9 & 6 & 0.999614 & 0.694538 & 0.114985 \\
\hline 10 & 6 & 2.295703 & 1.086053 & 0.154450 \\
\hline
\end{tabular}




$\begin{array}{rrrrrr}11 & 6 & 0 & 3.331559 & 0.062320 & 0.021670 \\ 12 & 7 & 0 & 2.835288 & -1.240724 & -0.141556 \\ 13 & 6 & 0 & 1.517179 & -1.660833 & -0.185428 \\ 14 & 6 & 0 & 2.725610 & 2.516504 & 0.316756 \\ 15 & 8 & 0 & 4.541249 & 0.264020 & 0.041114 \\ 16 & 8 & 0 & 1.207068 & -2.835962 & -0.330499 \\ 17 & 8 & 0 & -3.709713 & -1.595691 & 0.529194 \\ 18 & 1 & 0 & -3.885063 & 2.110113 & -0.644173 \\ 19 & 1 & 0 & -2.479836 & 2.168024 & -1.744479 \\ 20 & 1 & 0 & -3.455367 & -0.093260 & -1.472285 \\ 21 & 1 & 0 & -0.887043 & -1.995345 & -0.384741 \\ 22 & 1 & 0 & 0.182548 & 1.405107 & 0.181736 \\ 23 & 1 & 0 & 3.347464 & 2.639299 & 1.210020 \\ 24 & 1 & 0 & 3.333479 & 2.841448 & -0.534449 \\ 25 & 1 & 0 & 1.858623 & 3.177776 & 0.400619 \\ 26 & 1 & 0 & 3.525602 & -1.976471 & -0.241471 \\ 27 & 1 & 0 & -3.577231 & 0.334522 & 1.306652 \\ 28 & 1 & 0 & -1.221026 & 0.236704 & 1.678431 \\ 29 & 1 & 0 & -1.623224 & -1.490704 & 1.868870 \\ 30 & 1 & 0 & -2.091338 & 3.325670 & 0.265328 \\ 31 & 1 & 0 & -4.607808 & -1.442949 & 0.207276 \\ ---------------------------------------------------------\end{array}$

8. syn-dThd

\begin{tabular}{|c|c|c|c|c|c|}
\hline \multirow{2}{*}{$\begin{array}{l}\text { Center } \\
\text { Number }\end{array}$} & \multirow{2}{*}{$\begin{array}{l}\text { Atomic } \\
\text { Number }\end{array}$} & \multirow{2}{*}{\multicolumn{2}{|c|}{$\begin{array}{l}\text { Atomic } \\
\text { Type }\end{array}$}} & \multicolumn{2}{|c|}{ Coordinates (Angstroms } \\
\hline & & & & $\begin{array}{ll}X & Y\end{array}$ & $\mathrm{Z}$ \\
\hline 1 & 6 & 0 & 0.893407 & -1.016671 & -0.268286 \\
\hline 2 & 7 & 0 & 0.741225 & 0.134099 & 0.497562 \\
\hline 3 & 6 & 0 & 1.810464 & 1.004553 & 0.678720 \\
\hline 4 & 6 & 0 & 3.060585 & 0.800855 & 0.203141 \\
\hline 5 & 6 & 0 & 3.308361 & -0.411653 & -0.575947 \\
\hline 6 & 7 & 0 & 2.173819 & -1.225295 & -0.736806 \\
\hline 7 & 6 & 0 & -0.586572 & 0.533647 & 0.997311 \\
\hline 8 & 6 & 0 & -1.380929 & -0.522158 & 1.812720 \\
\hline 9 & 6 & 0 & -2.691222 & -0.723677 & 1.018303 \\
\hline 10 & 6 & 0 & -2.765979 & 0.587844 & 0.207044 \\
\hline 11 & 8 & 0 & -1.393565 & 0.911494 & -0.098773 \\
\hline 12 & 6 & 0 & -3.576653 & 0.513006 & -1.069207 \\
\hline 13 & 8 & 0 & -3.813780 & 1.847109 & -1.524435 \\
\hline 14 & 1 & 0 & -3.557066 & -0.810004 & 1.681683 \\
\hline 15 & 6 & 0 & 4.200447 & 1.753401 & 0.424349 \\
\hline 16 & 8 & 0 & 4.379146 & -0.740101 & -1.070159 \\
\hline 17 & 8 & 0 & -0.010824 & -1.812846 & $5-0.511963$ \\
\hline 18 & 1 & 0 & -4.522398 & -0.001894 & -0.848936 \\
\hline 19 & 1 & 0 & -3.028592 & -0.076376 & $5-1.812323$ \\
\hline 20 & 1 & 0 & -4.197508 & 1.811931 & -2.409110 \\
\hline 21 & 1 & 0 & -3.186054 & 1.388286 & 0.837976 \\
\hline 22 & 8 & 0 & -2.697032 & -1.887089 & 0.215821 \\
\hline 23 & 1 & 0 & -1.592142 & -0.117868 & 2.807187 \\
\hline 24 & 1 & 0 & -0.381546 & 1.410302 & 1.624718 \\
\hline 25 & 1 & 0 & 1.567747 & 1.892040 & 1.252726 \\
\hline 26 & 1 & 0 & 2.297401 & -2.066934 & -1.289343 \\
\hline
\end{tabular}




$\begin{array}{cccccc}27 & 1 & 0 & 4.601487 & 2.107321 & -0.531061 \\ 28 & 1 & 0 & 3.882298 & 2.618792 & 1.012133 \\ 29 & 1 & 0 & 5.025407 & 1.258123 & 0.947158 \\ 30 & 1 & 0 & -0.842739 & -1.462364 & 1.939366 \\ 31 & 1 & 0 & -1.814621 & -1.976403 & -0.190284\end{array}$

9. anti-2dAP

\begin{tabular}{|c|c|c|c|c|c|}
\hline \multirow{2}{*}{$\begin{array}{l}\text { Center } \\
\text { Number }\end{array}$} & \multirow{2}{*}{$\begin{array}{l}\text { Atomic } \\
\text { Number }\end{array}$} & \multirow{2}{*}{\multicolumn{2}{|c|}{$\begin{array}{r}\text { Atomic } \\
\text { Type }\end{array}$}} & \multicolumn{2}{|c|}{ Coordinates (Angstroms) } \\
\hline & & & & X $\quad Y$ & Z \\
\hline 1 & 7 & 0 & 4.457004 & -2.117936 & -0.204501 \\
\hline 2 & 6 & 0 & 3.732155 & -0.956029 & -0.161159 \\
\hline 3 & 7 & 0 & 2.401315 & -1.072741 & 0.012125 \\
\hline 4 & 7 & 0 & 4.447001 & 0.193196 & -0.267178 \\
\hline 5 & 6 & 0 & 1.791036 & 0.105414 & 0.084027 \\
\hline 6 & 6 & 0 & 3.789923 & 1.351949 & -0.187189 \\
\hline 7 & 7 & 0 & 0.446751 & 0.362277 & 0.265126 \\
\hline 8 & 6 & 0 & 2.407911 & 1.370116 & -0.002982 \\
\hline 9 & 6 & 0 & 0.329597 & 1.750736 & 0.261558 \\
\hline 10 & 6 & 0 & -0.608599 & -0.628775 & 0.345196 \\
\hline 11 & 7 & 0 & 1.462610 & 2.383214 & 0.110528 \\
\hline 12 & 8 & 0 & -1.404988 & -0.552203 & -0.850390 \\
\hline 13 & 6 & 0 & -1.583224 & -0.447786 & 1.533329 \\
\hline 14 & 6 & 0 & -2.789852 & -0.633250 & -0.508528 \\
\hline 15 & 6 & 0 & -2.892076 & 0.010599 & 0.884807 \\
\hline 16 & 6 & 0 & -3.598242 & 0.070680 & -1.587787 \\
\hline 17 & 8 & 0 & -4.006200 & -0.422774 & 1.641463 \\
\hline 18 & 8 & 0 & -4.955965 & 0.074456 & -1.123568 \\
\hline 19 & 1 & 0 & 5.402506 & -2.052069 & -0.548087 \\
\hline 20 & 1 & 0 & 3.951994 & -2.976044 & -0.362105 \\
\hline 21 & 1 & 0 & 4.375223 & 2.264602 & -0.274683 \\
\hline 22 & 1 & 0 & -0.634268 & 2.228452 & 0.372484 \\
\hline 23 & 1 & 0 & -0.092724 & -1.592141 & 0.376997 \\
\hline 24 & 1 & 0 & -1.763209 & -1.410392 & 2.021495 \\
\hline 25 & 1 & 0 & -1.197190 & 0.244336 & 2.284087 \\
\hline 26 & 1 & 0 & -3.113334 & -1.683122 & -0.431626 \\
\hline 27 & 1 & 0 & -2.909416 & 1.106724 & 0.772336 \\
\hline 28 & 1 & 0 & -3.222135 & 1.094851 & -1.715825 \\
\hline 29 & 1 & 0 & -3.506806 & -0.460992 & -2.542854 \\
\hline 30 & 1 & 0 & -4.799875 & -0.228507 & 1.119226 \\
\hline 31 & 1 & 0 & -5.528079 & 0.470471 & -1.791815 \\
\hline
\end{tabular}

10. syn-2dAP

\begin{tabular}{|c|c|c|c|}
\hline Center & Atomic & Atomic & Coordinates (Angstroms \\
\hline Number & Number & r Type & $\begin{array}{lll}X & Y & Z\end{array}$ \\
\hline 1 & 6 & -3.636435 & $\begin{array}{ll}-0.464116 & -0.012729\end{array}$ \\
\hline 2 & 6 & -2.470938 & -1.226317 \\
\hline 3 & 6 & -1.260830 & $-0.507391-0.003579$ \\
\hline
\end{tabular}




$\begin{array}{cccccc}4 & 7 & 0 & -1.158001 & 0.815940 & -0.137464 \\ 5 & 6 & 0 & -2.352145 & 1.443026 & -0.202626 \\ 6 & 7 & 0 & -3.576464 & 0.860602 & -0.152697 \\ 7 & 7 & 0 & -2.225761 & -2.584729 & 0.228449 \\ 8 & 6 & 0 & -0.923765 & -2.672053 & 0.244905 \\ 9 & 7 & 0 & -0.262822 & -1.453558 & 0.095033 \\ 10 & 6 & 0 & 1.180336 & -1.265620 & 0.092279 \\ 11 & 6 & 0 & 1.756356 & -0.634160 & -1.184562 \\ 12 & 6 & 0 & 3.013759 & 0.059996 & -0.669073 \\ 13 & 6 & 0 & 2.614628 & 0.488271 & 0.762771 \\ 14 & 8 & 0 & 1.535917 & -0.391163 & 1.155420 \\ 15 & 1 & 0 & 1.990011 & -1.377718 & -1.949638 \\ 16 & 6 & 0 & 2.162946 & 1.944150 & 0.897160 \\ 17 & 8 & 0 & 1.222183 & 2.339720 & -0.083170 \\ 18 & 8 & 0 & 4.060145 & -0.920184 & -0.667353 \\ 19 & 1 & 0 & -0.360034 & -3.589142 & 0.357887 \\ 20 & 1 & 0 & 3.037750 & 2.595923 & 0.780823 \\ 21 & 1 & 0 & 1.771617 & 2.088063 & 1.915395 \\ 22 & 1 & 0 & 0.414286 & 1.780212 & -0.006800 \\ 23 & 1 & 0 & 3.450481 & 0.314621 & 1.455512 \\ 24 & 1 & 0 & 3.292593 & 0.923146 & -1.283495 \\ 25 & 1 & 0 & 4.877081 & -0.517109 & -0.345358 \\ 26 & 1 & 0 & 1.060689 & 0.102643 & -1.590182 \\ 27 & 1 & 0 & 1.609465 & -2.260273 & 0.265988 \\ 28 & 1 & 0 & -4.624826 & -0.916156 & 0.029915 \\ 29 & 7 & 0 & -2.334224 & 2.800714 & -0.309253 \\ 30 & 1 & 0 & -1.461515 & 3.282919 & -0.458258 \\ 31 & 1 & 0 & -3.206123 & 3.278394 & -0.467780\end{array}$

11. anti-8-oxo-dGuo

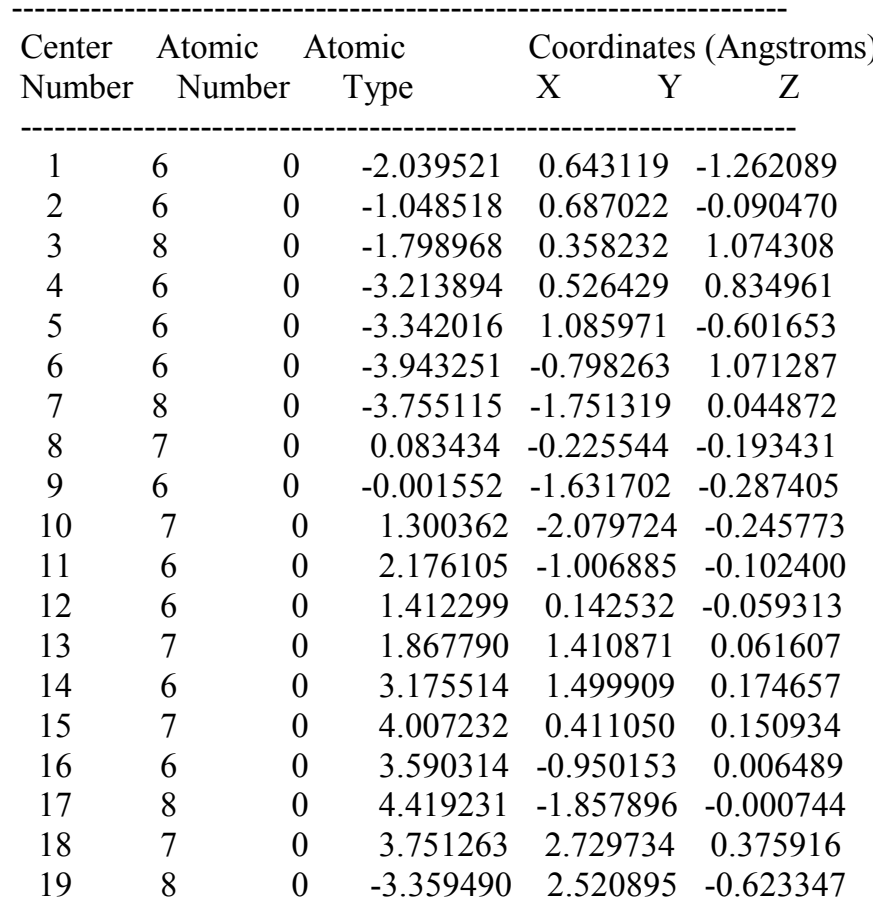




\begin{tabular}{|c|c|c|c|c|c|}
\hline 20 & 1 & 0 & -5.021172 & -0.598316 & 1.117626 \\
\hline 21 & 1 & 0 & -3.633553 & -1.188358 & 2.052530 \\
\hline 22 & 1 & 0 & -3.584263 & 1.264853 & 1.561069 \\
\hline 23 & 1 & 0 & -0.603302 & 1.680453 & 0.017404 \\
\hline 24 & 1 & 0 & 5.004030 & 0.521547 & 0.298061 \\
\hline 25 & 1 & 0 & 4.695479 & 2.885114 & 0.052804 \\
\hline 26 & 1 & 0 & 3.119032 & 3.507231 & 0.243266 \\
\hline 27 & 1 & 0 & -4.222582 & 0.681841 & -1.112509 \\
\hline 28 & 1 & 0 & -2.141087 & -0.374989 & -1.641272 \\
\hline 29 & 1 & 0 & -1.751173 & 1.314217 & -2.074272 \\
\hline 30 & 1 & 0 & -2.813888 & -2.010730 & 0.005803 \\
\hline 31 & 1 & 0 & -4.171490 & 2.835281 & -0.204723 \\
\hline 32 & 8 & 0 & -1.016532 & -2.316614 & -0.392806 \\
\hline 33 & 1 & 0 & 1.547499 & -3.057108 & -0.283197 \\
\hline
\end{tabular}

\section{2. syn-8-oxo-dGuo}

\begin{tabular}{|c|c|c|c|c|c|}
\hline \multirow{2}{*}{$\begin{array}{l}\text { Center } \\
\text { Number }\end{array}$} & \multirow{2}{*}{$\begin{array}{l}\text { Atomic } \\
\text { Number }\end{array}$} & \multirow{2}{*}{\multicolumn{2}{|c|}{$\begin{array}{l}\text { Atomic } \\
\text { Type }\end{array}$}} & \multicolumn{2}{|c|}{ Coordinates (Angstroms } \\
\hline & & & & $\begin{array}{ll}X & Y\end{array}$ & Z \\
\hline 1 & 6 & 0 & -1.987899 & -0.567083 & 1.211570 \\
\hline 2 & 6 & 0 & -1.348639 & -1.143732 & -0.060566 \\
\hline 3 & 8 & 0 & -1.738255 & -0.264233 & -1.117375 \\
\hline 4 & 6 & 0 & -2.925905 & 0.470227 & -0.756235 \\
\hline 5 & 6 & 0 & -3.288425 & 0.037243 & 0.688150 \\
\hline 6 & 7 & 0 & 0.100827 & -1.238886 & -0.039123 \\
\hline 7 & 6 & 0 & 1.030607 & -0.219778 & 0.039372 \\
\hline 8 & 6 & 0 & 2.287954 & -0.789309 & -0.013156 \\
\hline 9 & 7 & 0 & 2.119328 & -2.165729 & -0.137526 \\
\hline 10 & 6 & 0 & 0.775023 & -2.483898 & -0.172093 \\
\hline 11 & 7 & 0 & 0.762578 & 1.105044 & 0.153303 \\
\hline 12 & 6 & 0 & 1.826204 & 1.884921 & 0.193354 \\
\hline 13 & 7 & 0 & 3.101609 & 1.394211 & 0.153176 \\
\hline 14 & 6 & 0 & 3.458961 & 0.010626 & 0.039652 \\
\hline 15 & 8 & 0 & 4.641862 & -0.320361 & -0.001584 \\
\hline 16 & 7 & 0 & 1.658231 & 3.241501 & 0.224736 \\
\hline 17 & 6 & 0 & -2.653685 & 1.964231 & -0.933524 \\
\hline 18 & 8 & 0 & -1.707654 & 2.469381 & -0.004393 \\
\hline 19 & 8 & 0 & -4.274684 & -1.000957 & 0.708823 \\
\hline 20 & 1 & 0 & -2.175696 & -1.333635 & 1.966331 \\
\hline 21 & 1 & 0 & 2.381636 & 3.826431 & 0.615155 \\
\hline 22 & 1 & 0 & 0.706565 & 3.563813 & 0.349961 \\
\hline 23 & 1 & 0 & -3.585129 & 2.522202 & -0.779869 \\
\hline 24 & 1 & 0 & -2.325487 & 2.135470 & -1.969548 \\
\hline 25 & 1 & 0 & -0.899062 & 1.912953 & -0.051739 \\
\hline 26 & 1 & 0 & -3.732011 & 0.174601 & -1.443708 \\
\hline 27 & 1 & 0 & -3.618150 & 0.892585 & 1.289142 \\
\hline 28 & 1 & 0 & 2767 & -0.655459 & 0.374397 \\
\hline 29 & 1 & 0 & -1.353903 & 0.214876 & 1.635190 \\
\hline 30 & 1 & 0 & -1.694771 & -2.162431 & -0.259690 \\
\hline 31 & 1 & 0 & 3.894700 & 2.024979 & 0.128612 \\
\hline 32 & 8 & 0 & 0.256138 & -3.583250 & -0.277995 \\
\hline 33 & 1 & 0 & 2.851634 & -2.854706 & -0.215135 \\
\hline
\end{tabular}


13. anti-8-oxo-dAdo

\begin{tabular}{|c|c|c|c|c|c|}
\hline \multirow{2}{*}{$\begin{array}{l}\text { Center } \\
\text { Number }\end{array}$} & \multirow{2}{*}{\multicolumn{2}{|c|}{$\begin{array}{l}\text { Atomic } \\
\text { Number }\end{array}$}} & \multirow{2}{*}{$\begin{array}{l}\text { Atomic } \\
\text { Type }\end{array}$} & \multicolumn{2}{|c|}{ Coordinates (Angstroms) } \\
\hline & & & & $\begin{array}{ll}X & Y\end{array}$ & $\mathrm{Z}$ \\
\hline 1 & 6 & 0 & 1.563917 & 0.543606 & 0.012259 \\
\hline 2 & 7 & 0 & 0.293704 & 0.006544 & -0.167788 \\
\hline 3 & 6 & 0 & 0.394005 & -1.383908 & -0.309119 \\
\hline 4 & 7 & 0 & 1.753879 & -1.669986 & -0.286755 \\
\hline 5 & 6 & 0 & 2.485672 & -0.503445 & -0.062913 \\
\hline 6 & 7 & 0 & 1.872435 & 1.821417 & 0.210728 \\
\hline 7 & 6 & 0 & 3.192820 & 2.022152 & 0.344437 \\
\hline 8 & 7 & 0 & 4.168291 & 1.113047 & 0.273064 \\
\hline 9 & 6 & 0 & 3.836907 & -0.176033 & 0.054726 \\
\hline 10 & 6 & 0 & -0.948129 & 0.762550 & -0.047498 \\
\hline 11 & 8 & 0 & -1.668220 & 0.282744 & 1.083681 \\
\hline 12 & 6 & 0 & -3.089784 & 0.296745 & 0.826440 \\
\hline 13 & 6 & 0 & -3.265052 & 0.891254 & -0.591912 \\
\hline 14 & 6 & 0 & -1.905773 & 0.644950 & -1.240955 \\
\hline 15 & 6 & 0 & -3.659154 & -1.111900 & 1.003761 \\
\hline 16 & 8 & 0 & -3.324313 & -2.002742 & -0.042494 \\
\hline 17 & 8 & 0 & -3.466756 & 2.310600 & -0.562605 \\
\hline 18 & 7 & 0 & 4.856701 & -1.100903 & 0.018742 \\
\hline 19 & 1 & 0 & -4.754221 & -1.047191 & 1.019618 \\
\hline 20 & 1 & 0 & -3.332686 & -1.493227 & 1.982935 \\
\hline 21 & 1 & 0 & -3.553792 & 0.959794 & 1.571215 \\
\hline 22 & 1 & 0 & -0.632693 & 1.797399 & 0.115259 \\
\hline 23 & 1 & 0 & -4.077706 & 0.396940 & -1.135468 \\
\hline 24 & 1 & 0 & -1.871317 & -0.361333 & -1.662168 \\
\hline 25 & 1 & 0 & -1.691903 & 1.380646 & -2.019345 \\
\hline 26 & 1 & 0 & 4.729352 & -1.933970 & -0.537928 \\
\hline 27 & 1 & 0 & 5.781045 & -0.693060 & -0.036088 \\
\hline 28 & 1 & 0 & 3.507097 & 3.046531 & 0.522994 \\
\hline 29 & 1 & 0 & -4.317269 & 2.503969 & -0.147046 \\
\hline 30 & 1 & 0 & -2.357111 & -2.132500 & -0.063553 \\
\hline 31 & 8 & 0 & -0.509317 & -2.203830 & -0.436015 \\
\hline 32 & 1 & 0 & 2.072422 & -2.624839 & -0.222264 \\
\hline
\end{tabular}

14. syn-8-oxo-dAdo

\begin{tabular}{|c|c|c|c|c|}
\hline \multirow{2}{*}{$\begin{array}{l}\text { Center } \\
\text { Number }\end{array}$} & \multirow{2}{*}{$\begin{array}{l}\text { Atomic } \\
\text { Number }\end{array}$} & \multirow{2}{*}{$\begin{array}{l}\text { Atomic } \\
\text { Type }\end{array}$} & \multicolumn{2}{|c|}{ Coordinates (Angstroms } \\
\hline & & & X $\quad Y$ & $\mathrm{Z}$ \\
\hline 1 & 6 & 1.897988 & 0.533272 & 1.217437 \\
\hline 2 & 6 & 1.194942 & 1.049732 & -0.045379 \\
\hline 3 & 8 & 1.651946 & 0.222676 & -1.111650 \\
\hline 4 & 6 & 2.892528 & -0.426106 & -0.755205 \\
\hline 5 & 6 & 3.243068 & 0.062349 & 0.671740 \\
\hline 6 & 7 & -0.260162 & 1.005584 & -0.008432 \\
\hline 7 & 6 & -1.088006 & -0.103360 & 0.075296 \\
\hline
\end{tabular}




$\begin{array}{cccrrr}8 & 6 & 0 & -2.410346 & 0.346060 & -0.012648 \\ 9 & 7 & 0 & -2.365962 & 1.732729 & -0.145076 \\ 10 & 6 & 0 & -1.041843 & 2.164566 & -0.200663 \\ 11 & 7 & 0 & -0.741926 & -1.385624 & 0.209898 \\ 12 & 6 & 0 & -1.794154 & -2.222120 & 0.259096 \\ 13 & 7 & 0 & -3.085763 & -1.904806 & 0.209556 \\ 14 & 6 & 0 & -3.425998 & -0.604126 & 0.083041 \\ 15 & 6 & 0 & 2.728571 & -1.940662 & -0.888547 \\ 16 & 8 & 0 & 1.919244 & -2.514984 & 0.119530 \\ 17 & 8 & 0 & 4.112285 & 1.202102 & 0.655983 \\ 18 & 1 & 0 & 2.019543 & 1.311913 & 1.973598 \\ 19 & 1 & 0 & 3.716445 & -2.410889 & -0.806423 \\ 20 & 1 & 0 & 2.338693 & -2.156620 & -1.894770 \\ 21 & 1 & 0 & 1.014372 & -2.138966 & 0.064528 \\ 22 & 1 & 0 & 3.661319 & -0.089564 & -1.466109 \\ 23 & 1 & 0 & 3.674516 & -0.743908 & 1.275244 \\ 24 & 1 & 0 & 4.972010 & 0.943805 & 0.298707 \\ 25 & 1 & 0 & 1.351748 & -0.311256 & 1.642477 \\ 26 & 1 & 0 & 1.437824 & 2.098380 & -0.241705 \\ 27 & 8 & 0 & -0.646005 & 3.304389 & -0.366470 \\ 28 & 1 & 0 & -3.114634 & 2.349695 & -0.420470 \\ 29 & 7 & 0 & -4.765630 & -0.302231 & -0.000244 \\ 30 & 1 & 0 & -5.380382 & -1.069775 & 0.237328 \\ 31 & 1 & 0 & -5.076174 & 0.604748 & 0.317179 \\ 32 & 1 & 0 & -1.559433 & -3.278221 & 0.356338 \\ -----------------------------------------------------\end{array}$

15. anti-2'-dI

\begin{tabular}{|c|c|c|c|c|c|}
\hline \multirow{2}{*}{$\begin{array}{l}\text { Center } \\
\text { Number }\end{array}$} & \multirow{2}{*}{\multicolumn{2}{|c|}{$\begin{array}{l}\text { Atomic } \\
\text { Number }\end{array}$}} & \multirow{2}{*}{$\begin{array}{l}\text { Atomic } \\
\text { Type }\end{array}$} & \multicolumn{2}{|c|}{ Coordinates (Angstroms } \\
\hline & & & & $\mathrm{X}$ & $\mathrm{Z}$ \\
\hline 1 & 6 & 0 & 1.869573 & -0.645873 & -1.198530 \\
\hline 2 & 6 & 0 & 0.952225 & -0.696876 & 0.033526 \\
\hline 3 & 8 & 0 & 1.717449 & -0.157531 & 1.114833 \\
\hline 4 & 6 & 0 & 3.108869 & -0.075874 & 0.775510 \\
\hline 5 & 6 & 0 & 3.263608 & -0.778315 & -0.595472 \\
\hline 6 & 6 & 0 & 3.580110 & 1.373730 & 0.814762 \\
\hline 7 & 8 & 0 & 2.993440 & 2.096887 & -0.270294 \\
\hline 8 & 7 & 0 & -0.275051 & 0.070959 & -0.101225 \\
\hline 9 & 6 & 0 & -0.399585 & 1.432867 & -0.318583 \\
\hline 10 & 7 & 0 & -1.646542 & 1.842194 & -0.342660 \\
\hline 11 & 6 & 0 & -2.391030 & 0.703288 & -0.126699 \\
\hline 12 & 6 & 0 & -1.558675 & -0.406720 & 0.034193 \\
\hline 13 & 7 & 0 & -1.911879 & -1.702158 & 0.266811 \\
\hline 14 & 6 & 0 & -3.203363 & -1.861312 & 0.343793 \\
\hline 15 & 7 & 0 & -4.114280 & -0.853582 & 0.203509 \\
\hline 16 & 6 & 0 & -3.821976 & 0.527312 & -0.046818 \\
\hline 17 & 8 & 0 & -4.735161 & 1.332166 & -0.154636 \\
\hline 18 & 8 & 0 & 3.534243 & -2.176015 & -0.452408 \\
\hline 19 & 1 & 0 & 4.678586 & 1.389702 & 0.742316 \\
\hline 20 & 1 & 0 & 3.286022 & 1.807616 & 1.779160 \\
\hline 21 & 1 & 0 & 3.674093 & -0.624600 & 1.542055 \\
\hline 22 & 1 & 0 & 0.642717 & -1.717639 & 0.273069 \\
\hline
\end{tabular}




$\begin{array}{rrrrrr}23 & 1 & 0 & 0.475825 & 2.057245 & -0.427821 \\ 24 & 1 & 0 & -5.103661 & -1.062847 & 0.275670 \\ 25 & 1 & 0 & 4.031568 & -0.298438 & -1.213750 \\ 26 & 1 & 0 & 1.774456 & 0.319051 & -1.700416 \\ 27 & 1 & 0 & 1.657347 & -1.448391 & -1.907843 \\ 28 & 1 & 0 & 3.235719 & 3.028470 & -0.200024 \\ 29 & 1 & 0 & 4.414063 & -2.298504 & -0.072254 \\ 30 & 1 & 0 & -3.610761 & -2.850974 & 0.530737\end{array}$

16. syn-2'-dI

\begin{tabular}{|c|c|c|c|c|c|}
\hline \multirow{2}{*}{$\begin{array}{l}\text { Center } \\
\text { Number }\end{array}$} & Atomic & \multirow{2}{*}{\multicolumn{2}{|c|}{$\begin{array}{l}\text { Atomic } \\
\text { Type }\end{array}$}} & \multicolumn{2}{|c|}{ Coordinates (Angstroms } \\
\hline & & & & $\mathrm{X}$ & $\mathrm{Z}$ \\
\hline 1 & 6 & 0 & 1.822924 & -0.698763 & -1.165230 \\
\hline 2 & 6 & 0 & 1.165965 & -1.182732 & 0.134906 \\
\hline 3 & 8 & 0 & 1.625494 & -0.306194 & 1.152943 \\
\hline 4 & 6 & 0 & 2.842187 & 0.360446 & 0.739941 \\
\hline 5 & 6 & 0 & 3.172369 & -0.187367 & -0.669387 \\
\hline 6 & 7 & 0 & -0.291990 & -1.190020 & 0.135749 \\
\hline 7 & 6 & 0 & -1.170894 & -0.140278 & -0.020185 \\
\hline 8 & 6 & 0 & -2.446170 & -0.703944 & 0.069161 \\
\hline 9 & 7 & 0 & -2.369192 & -2.060472 & 0.282160 \\
\hline 10 & 6 & 0 & -1.083329 & -2.309523 & 0.322780 \\
\hline 11 & 7 & 0 & -0.857433 & 1.176601 & -0.211985 \\
\hline 12 & 6 & 0 & -1.907719 & 1.944232 & -0.325370 \\
\hline 13 & 7 & 0 & -3.191906 & 1.499289 & -0.260789 \\
\hline 14 & 6 & 0 & -3.611390 & 0.142677 & -0.055194 \\
\hline 15 & 8 & 0 & -4.800214 & -0.125678 & -0.013058 \\
\hline 16 & 6 & & 2.636167 & 1.873790 & 0.810638 \\
\hline 17 & 8 & 0 & 1.781859 & 2.377998 & -0.198575 \\
\hline 18 & 8 & 0 & 4.051250 & -1.318440 & -0.615426 \\
\hline 19 & 1 & 0 & 1.936853 & -1.498000 & -1.900640 \\
\hline 20 & 1 & 0 & -0.638570 & -3.282953 & $3 \quad 0.481297$ \\
\hline 21 & 1 & 0 & 3.606126 & 2.368709 & 0.679217 \\
\hline 22 & 1 & 0 & 2.267257 & 2.123117 & 1.816704 \\
\hline 23 & 1 & 0 & 0.890231 & 1.982772 & -0.098995 \\
\hline 24 & 1 & 0 & 3.632945 & 0.077081 & 1.449048 \\
\hline 25 & 1 & 0 & 3.583813 & 0.593490 & -1.318212 \\
\hline 26 & 1 & 0 & 4.923195 & -1.034326 & -0.311210 \\
\hline 27 & 1 & 0 & 1.247547 & 0.121381 & -1.598593 \\
\hline 28 & 1 & 0 & 1.462355 & -2.214344 & 0.362104 \\
\hline 29 & 1 & 0 & -3.953778 & 2.161872 & -0.356086 \\
\hline 30 & 1 & 0 & -1.772109 & 3.010704 & -0.480007 \\
\hline
\end{tabular}

17. anti-5-methyl-2'-dCyd

\begin{tabular}{|c|c|c|c|}
\hline Center & Atomic & Atomic & Coordinates (Angstroms) \\
\hline Number & Number & Type & $\mathrm{X}$ \\
\hline 1 & 6 & -1.637832 & -0.763735 \\
\hline
\end{tabular}




$\begin{array}{cccrrc}2 & 6 & 0 & -0.843007 & -0.919601 & -0.211402 \\ 3 & 8 & 0 & -1.445628 & -0.002626 & -1.133363 \\ 4 & 6 & 0 & -2.759229 & 0.372505 & -0.706334 \\ 5 & 6 & 0 & -3.037835 & -0.404695 & 0.606030 \\ 6 & 6 & 0 & -2.844443 & 1.896869 & -0.603403 \\ 7 & 8 & 0 & -2.003048 & 2.455390 & 0.410622 \\ 8 & 7 & 0 & 0.580132 & -0.600867 & -0.102119 \\ 9 & 6 & 0 & 0.983922 & 0.695667 & 0.028532 \\ 10 & 6 & 0 & 2.301102 & 1.037740 & 0.101017 \\ 11 & 6 & 0 & 3.220107 & -0.073151 & 0.015185 \\ 12 & 7 & 0 & 2.847861 & -1.334975 & -0.092833 \\ 13 & 6 & 0 & 1.525080 & -1.678866 & -0.135091 \\ 14 & 7 & 0 & 4.563236 & 0.167950 & 0.017534 \\ 15 & 8 & 0 & 1.112776 & -2.834320 & -0.187987 \\ 16 & 8 & 0 & -3.737749 & -1.627856 & 0.375580 \\ 17 & 1 & 0 & -3.891454 & 2.198238 & -0.455527 \\ 18 & 1 & 0 & -2.501120 & 2.324401 & -1.549698 \\ 19 & 1 & 0 & -3.477198 & 0.054849 & -1.478610 \\ 20 & 1 & 0 & -0.889384 & -1.939607 & -0.594119 \\ 21 & 1 & 0 & 0.190198 & 1.435583 & 0.053767 \\ 22 & 1 & 0 & 4.935370 & 1.068938 & 0.266257 \\ 23 & 1 & 0 & 5.173849 & -0.634951 & 0.054534 \\ 24 & 1 & 0 & -3.591419 & 0.208778 & 1.332486 \\ 25 & 1 & 0 & -1.220815 & 0.055729 & 1.687207 \\ 26 & 1 & 0 & -1.637605 & -1.676967 & 1.692582 \\ 27 & 1 & 0 & -2.473385 & 2.468291 & 1.253159 \\ 28 & 1 & 0 & -4.604341 & -1.440374 & -0.008930 \\ 29 & 6 & 0 & 2.760216 & 2.466724 & 0.226344 \\ 30 & 1 & 0 & 3.339626 & 2.636447 & 1.144241 \\ 31 & 1 & 0 & 3.387646 & 2.770522 & -0.621829 \\ 32 & 1 & 0 & 1.902130 & 3.143919 & 0.256431 \\ -----------------------------------\end{array}$

18. syn-5-methyl-2'-dCyd

\begin{tabular}{|c|c|c|c|c|}
\hline \multirow{2}{*}{$\begin{array}{l}\text { Center } \\
\text { Number }\end{array}$} & \multirow{2}{*}{$\begin{array}{l}\text { Atomic } \\
\text { Number }\end{array}$} & \multirow{2}{*}{$\begin{array}{l}\text { Atomic } \\
\text { Type }\end{array}$} & \multicolumn{2}{|c|}{ Coordinates (Angstroms } \\
\hline & & & $\begin{array}{ll}X & Y\end{array}$ & $\mathrm{Z}$ \\
\hline 1 & 6 & -1.831737 & -1.223178 & 0.113402 \\
\hline 2 & 7 & -0.711694 & -0.462227 & -0.074252 \\
\hline 3 & 6 & -0.830863 & 0.953996 & -0.228402 \\
\hline 4 & 7 & -2.086883 & 1.476805 & -0.262224 \\
\hline 5 & 6 & -3.155217 & 0.714405 & -0.111138 \\
\hline 6 & 6 & -3.092846 & -0.710515 & 0.103443 \\
\hline 7 & 6 & 0.602019 & -1.127294 & -0.009418 \\
\hline 8 & 6 & 1.552690 & -0.897769 & -1.185572 \\
\hline 9 & 6 & 2.943259 & -1.049961 & -0.532381 \\
\hline 10 & 6 & 2.702833 & -0.589801 & 0.940390 \\
\hline 11 & 8 & 1.278775 & -0.711830 & 1.165644 \\
\hline 12 & 6 & 3.165538 & 0.836686 & 1.238768 \\
\hline 13 & 8 & 3.984229 & -0.367932 & -1.197796 \\
\hline 14 & 8 & 0.181890 & 1.649765 & -0.337648 \\
\hline 15 & 7 & -4.363304 & 1.334970 & -0.180949 \\
\hline 16 & 1 & 3.198175 & -1.264833 & 1.651123 \\
\hline
\end{tabular}




$\begin{array}{rrrrrr}17 & 1 & 0 & 3.240949 & -2.103961 & -0.534229 \\ 18 & 1 & 0 & 0.363234 & -2.196856 & 0.075927 \\ 19 & 1 & 0 & -1.657425 & -2.283112 & 0.269331 \\ 20 & 1 & 0 & -4.366640 & 2.342024 & -0.250075 \\ 21 & 1 & 0 & -5.220085 & 0.860184 & 0.048305 \\ 22 & 1 & 0 & 1.440206 & 0.110782 & -1.584216 \\ 23 & 1 & 0 & 1.386818 & -1.620070 & -1.989322 \\ 24 & 1 & 0 & 4.253729 & 0.834543 & 1.359745 \\ 25 & 8 & 0 & 2.878528 & 1.758995 & 0.194740 \\ 26 & 1 & 0 & 2.717579 & 1.156214 & 2.189875 \\ 27 & 1 & 0 & 1.906377 & 1.818695 & 0.079512 \\ 28 & 1 & 0 & 3.790216 & 0.585095 & -1.109935 \\ 29 & 6 & 0 & -4.318838 & -1.562154 & 0.298149 \\ 30 & 1 & 0 & -4.894717 & -1.257228 & 1.181852 \\ 31 & 1 & 0 & -4.990058 & -1.515800 & -0.569005 \\ 32 & 1 & 0 & -4.043588 & -2.610994 & 0.440121 \\ ---------------------------------------------------------------\end{array}$

V. Cartesian Coordinates of the Modified syn-dGuo Where the 5'-OH Hydrogen Bond has been Manually Rotated to Break the Intramolecular Hydrogen Bond Formation with N(3) of the Base

\begin{tabular}{|c|c|c|c|c|c|}
\hline \multirow{2}{*}{$\begin{array}{l}\text { Center } \\
\text { Number }\end{array}$} & \multirow{2}{*}{$\begin{array}{l}\text { Atomic } \\
\text { Number }\end{array}$} & \multirow{2}{*}{$\begin{array}{l}\text { Atomic } \\
\text { Type }\end{array}$} & \multicolumn{3}{|c|}{ Coordinates (Angstroms) } \\
\hline & & & $\mathrm{X}$ & $\mathrm{Y}$ & Z \\
\hline \multicolumn{6}{|c|}{ 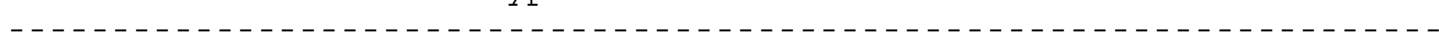 } \\
\hline 1 & 6 & 0 & 1.916105 & -0.685917 & -1.195259 \\
\hline 2 & 6 & 0 & 1.312590 & -1.273036 & 0.078326 \\
\hline 3 & 8 & 0 & 1.726845 & -0.423044 & 1.106506 \\
\hline 4 & 6 & 0 & 2.916930 & 0.268863 & 0.762165 \\
\hline 5 & 6 & 0 & 3.242526 & -0.143880 & -0.687789 \\
\hline 6 & 7 & 0 & -0.126654 & -1.388556 & 0.096157 \\
\hline 7 & 6 & 0 & -1.073898 & -0.418090 & -0.005249 \\
\hline 8 & 6 & 0 & -2.277975 & -1.062263 & 0.079996 \\
\hline 9 & 7 & 0 & -2.093962 & -2.415175 & 0.245997 \\
\hline 10 & 6 & 0 & -0.824769 & -2.565797 & 0.256599 \\
\hline 11 & 7 & 0 & -0.851036 & 0.914248 & -0.163663 \\
\hline 12 & 6 & 0 & -1.934629 & 1.616023 & -0.227312 \\
\hline 13 & 7 & 0 & -3.178388 & 1.080401 & -0.159948 \\
\hline 14 & 6 & 0 & -3.486973 & -0.290429 & 0.006570 \\
\hline 15 & 8 & 0 & -4.633199 & -0.622856 & 0.064920 \\
\hline 16 & 7 & 0 & -1.857545 & 2.966304 & -0.330611 \\
\hline 17 & 6 & 0 & 2.693600 & 1.756992 & 0.973497 \\
\hline 18 & 8 & 0 & 1.763468 & 2.294624 & 0.082345 \\
\hline 19 & 8 & 0 & 4.171256 & -1.202886 & -0.729043 \\
\hline 20 & 1 & 0 & 2.052171 & -1.426377 & -1.972228 \\
\hline 21 & 1 & 0 & -0.310049 & -3.498043 & 0.372911 \\
\hline 22 & 1 & 0 & -2.624655 & 3.470343 & -0.711352 \\
\hline 23 & 1 & 0 & -0.953469 & 3.336827 & -0.521835 \\
\hline 24 & 1 & 0 & 3.628273 & 2.285948 & 0.817756 \\
\hline 25 & 1 & 0 & 2.386275 & 1.916531 & 2.004362 \\
\hline 26 & 1 & 0 & 2.101044 & 2.991201 & -0.470942 \\
\hline 27 & 1 & 0 & 3.709769 & -0.063701 & 1.427100 \\
\hline 28 & 1 & 0 & 3.598294 & 0.694820 & -1.274498 \\
\hline 29 & 1 & 0 & 5.027060 & -0.913306 & -0.457704 \\
\hline 30 & 1 & 0 & 1.297157 & 0.119106 & -1.565816 \\
\hline 31 & 1 & 0 & 1.689333 & -2.274134 & 0.251491 \\
\hline
\end{tabular}




\section{References to Supporting Information}

(1) Murov, S. L.; Hug, G. L.; Carmichael, I. Handbook of Photochemistry, 2nd ed. ed.; Marcel Dekker, Inc.: New York, 1993.

(2) Seidel, C. A. M.; Schulz, A.; Sauer, M. H. M. J. Phys. Chem. 1996, 100, 5541. 